\title{
Energy Circulation Theory to Derive the Cosmic Evolution, Electric Charge, Light and Electromagnetism
}

\author{
Shigeto Nagao \\ Ikeda, Osaka, Japan \\ snagao@lilac.plala.or.jp \\ Received 19 July 2018 \\ Accepted 7 October 2018 \\ Published 5 November 2018
}

\begin{abstract}
Starting from the premise that the energy is vibrations in multiple dimensions, we propose the following energy circulation theory: Energy is the movement of an intrinsic energy, and can work as a new intrinsic energy for the direction perpendicular to the original movement. The fundamental force works between two momentums, by which antiparallel energy movements bend and make a circle. A form of the fundamental force is the electric force working on the electric charge, which is the momentum in a hidden dimension. Simultaneous separations of two pairs of conjugate energy circulations provide two universes, where the energy distribution is expanding in four dimensions. We divide the energy into two parts. The space energy is a rest energy corresponding to the vacuum space. The apparent energy is the kinetic energy and what we detect as energy in the 3D space. We induce the electric charge, light and electromagnetism from an energy circulation in one hidden and one space dimensions.
\end{abstract}

Keywords: Cosmology; energy circulation theory; fundamental force; 4D spherical universe; space energy; apparent energy; electric charge; electric force.

\section{Introduction}

It was striking that Perlmutter et al. reported from their observed data of type Ia supernovae that the expansion of the universe is accelerating in spite of the gravitational attraction. ${ }^{1-4}$ Dark energy resulting from an unknown repulsive force is widely expected. However, no candidate for the energy or the force is yet fully established. The interpretation of the acceleration in the expansion is subject to the invariant light speed during the whole expansion period. Would the light speed invariance be duly true with an absolute experimental proof? Those evidences for the

This is an Open Access article published by World Scientific Publishing Company. It is distributed under the terms of the Creative Commons Attribution 4.0 (CC-BY) License. Further distribution of this work is permitted, provided the original work is properly cited. 
theory of relativity, which are said to have been experimentally proved, are subject to the light speed invariance or the rest-mass invariance by frame selection. We formerly reported that Michelson-Morley-type experiments are incapable to detect a shift in the location of an interference fringe because the combined two beams are in a common direction at the last stage from the half-mirror to the detector. ${ }^{5,6}$ If we do not stick to the invariances, such evidences may show a variation in the light speed or the rest mass by frame selection. There is another mystery why the current universe is matter-abundant with little antimatters. How could such an asymmetry of the universe be generated? On the other hand, the quantum mechanics, which is one of the greatest achievements of modern physics established in the twentieth century, can successfully explain the phenomena in particle physics and chemistry. It is generally said that the wave function of a quantum particle represents the probability of existence but is not a vibration of a real medium. This is called the Copenhagen interpretation. ${ }^{7}$ Even if we interpret the wave function as a vibration in a medium, the outcome of the quantum mechanics would not request a drastic change from the current one, we guess. Would it not be possible to define the quantization clearly?

We have been proposing the four-dimensional (4D) spherical model of the universe, in which the space energy spreads with expansion in a three-dimensional (3D) surface of a $4 \mathrm{D}$ sphere. ${ }^{5,6,8-10}$ The expected Hubble diagram from the model showed an excellent fit to the reported ones of observed supernovae for the present radius being about $70 \%$ of its maximum. ${ }^{8}$ It motivated us to investigate a potential scenario to provide the current universe without a critical conflict with the real observation and in a manner to once reset and newly define key concepts and terms starting from some premises. In this paper, we examine in detail how the universe evolves. We start from the premise that there exists the initial energy exhibiting vibrations in multiple dimensions. In order to exhibit vibrations, energy should circulate, for which a force is required. We will discuss what is a force, how to accelerate an energy movement, and propose a theory based on an energy circulation. Subsequently, we will see each generation of a $4 \mathrm{D}$ spherical universe, the electric charge, the light and so on.

As preceding notes to discussion, we raise key points on the Lorentz transformation, energy-mass equivalence and energy invariance. We previously reported in detail why the Michelson-Morley-type experiments ${ }^{11-13}$ are incapable to detect a shift in the location of an interference fringe. ${ }^{5,6}$ The phase of the parallel beam to the apparatus movement should be released $\Delta t$ earlier than that of the perpendicular beam in order to reach the detector simultaneously for interfering. The combined beam from the two split beams propagates from the half-mirror to the detector as shown by the following formula, where $x$ denotes the distance from the half-mirror in the direction to the detector. Because the direction is same for both component beams, the light speed is common for both even by the frame attached to the apparatus not only by the medium's frame. The wavenumber and the frequency are 
also common. The situation is same for such a modern experiment reported by Hermann et al.: ${ }^{13}$

$$
\begin{aligned}
\varphi_{\perp}+\varphi_{/ /}= & A \exp (i(k x-\omega t))+A \exp (i(k x-\omega(t+\Delta t))) \\
= & A \exp \left(i\left(k x-\omega t-\frac{\omega \Delta t}{2}+\frac{\omega \Delta t}{2}\right)\right) \\
& +A \exp \left(i\left(k x-\omega t-\frac{\omega \Delta t}{2}-\frac{\omega \Delta t}{2}\right)\right) \\
= & A\left(\exp \left(i \frac{\omega \Delta t}{2}\right)+\exp \left(-i \frac{\omega \Delta t}{2}\right)\right) \exp \left(i\left(k x-\omega t-\frac{\omega \Delta t}{2}\right)\right) \\
= & 2 A \cos \frac{\omega \Delta t}{2} \exp \left(i\left(k x-\omega t-\frac{\omega \Delta t}{2}\right)\right) .
\end{aligned}
$$

The combined beam passes through both the slits with a small distance, and projects an interference fringe on the screen. By variation of $\Delta t$ there is no change in the frequency or the wavenumber but only the amplitude and the phase vary. Therefore, there is no change in the location or the pattern of the fringe but the brightness alters by variation of $\Delta t$.

Thus the light speed invariance by frame selection has not yet been experimentally proved. We expect that latest Michelson interferometers for gravitational waves would be capable to detect an anisotropy of the light speed by the apparatus frame as a circadian rhythm in the brightness of the combined beam. According to the proposed $4 \mathrm{D}$ spherical model, the Lorentz transformation in the special relativity ${ }^{14}$ is invalid since there is the wave medium.

Another outcome from the special relativity is the energy-mass equivalence shown by $E=m c^{2} \cdot{ }^{15-17}$ This equation can be induced even without using the Lorentz transformation or the light speed invariance by frame selection, for instance from the conservation of momentum in a process where light is released from one end and captured at the other end. ${ }^{18}$ Thus the energy-mass equivalence should be valid.

The special relativity insisted the invariance of the $4 \mathrm{D}$ momentum in the Minkowski space-time and that the quantity $E^{2}-(p c)^{2}=\left(m c^{2}\right)^{2}$ is invariant from frame selection, while the energy $E$ and the momentum $p$ depend on the frame. ${ }^{16,17}$ The mass in the equation is called the rest mass or the invariant mass, which is conserved and invariant for all observers. ${ }^{16}$ We insist that the total energy should be conserved and invariant by selection of a frame, but its breakdown to the rest energy and the kinetic energy should depend on a frame to express the subject.

\section{Energy Circulation and the Fundamental Force}

In this paper we examine how the initial energy can evolve to the $4 \mathrm{D}$ spherical universe that we proposed. This paper shall overwrite all discrepancies in what were reported before. 


\subsection{Energy circulation theory}

We start from the premise that in the cosmos there exists the initial energy, which exhibits vibrations in multiple dimensions. In order to allow an energy vibration in plural dimensions, some underling conditions should be required. Let us see some factors.

What is the term "energy"? In standard physics, energy is described by various other physical properties such as the mass, velocity, acceleration, electric charge, field and so on. We may inversely define the energy first and then induce other physical properties. When we express that something exists physically, what property can exist? Can a property, which is not an energy, exist? We guess anything that exists should fall in energy. Let us define the term "energy" as what exists in the cosmos. There are various distributions and movements of energy. Those present other physical properties. We further guess that energy cannot be static but should be in motion, however we may treat a steady state of energy movement collectively as stationary. For energy movement, let us define the "intrinsic energy" so that its movement renders an additional energy. The movement of the intrinsic energy exhibits a new total energy. A potential form of a steady state of energy movement is as follows: An intrinsic energy lies even on a circumference of a circle and circulates by $\omega$ in a two-dimensional (2D) plane. We can express the energy circulation using the total energy $E$ instead of the intrinsic energy by a function as

$$
E \varphi=E \exp (i \omega t)=E(\cos \omega t+i \sin \omega t), \quad \mathbf{X}=\mu \varphi, \quad \mathbf{Y}=-i \mu \varphi \quad(\mu=\text { radius }) .
$$

If we treat the total energy collectively, we can regard $\varphi$ as similar to a wave function exhibiting the energy $\varphi^{*} E \varphi=E$ in standard quantum mechanics. However, the function here also shows that any local part of the intrinsic energy on the circumference is circulating at the velocity $\mu \omega$ as shown by $(X, Y)=(\mu \varphi,-i \mu \varphi)$. The function $\varphi$ indicates not only the distribution of the total energy but also the circulation of its intrinsic energy as an internal motion. We can also regard that the location of a local part of the intrinsic energy is fluctuating by $\varphi$ in one dimension within $X-Y$.

In order to provide an energy circulation, a centripetal force is required. What force works on the intrinsic energy or its movement? It is considerable that between conjugate circulations of energy, a force would work and attract them to each other. A pair of conjugate circulations of energy gives a one-dimensional vibration in total as shown below:

$$
\frac{E}{2}\left(\varphi+\varphi^{*}\right)=\frac{E}{2}(\exp (i \omega t)+\exp (-i \omega t))=E \cos \omega t
$$

Taking into consideration the above-mentioned, we propose potential underlying rules to enable an energy circulation and an energy vibration in multiple dimensions. These are not theoretical derivations but rather are hypotheses, which we collectively 
call a proposed theory. Here we propose the following "energy circulation theory" (ECT).

- A movement of an intrinsic energy vests an additional energy and exhibits a new total energy. The total energy can work as a new intrinsic energy for further movement in a perpendicular direction to the original movement. If an energy is static in a direction, it serves as the "rest energy" for the direction. The rest energy depends on the target direction.

- The location of total energy is equal to that of its intrinsic energy. If an intrinsic energy is moving at a speed $v$, the location of the resulted total energy is also moving at $v$.

- Take two energies $\left(E_{1}, E_{2}\right)$, which are derived from a movement of an intrinsic energy in a common plane. Call the direction between their positions as the "distance direction". We will examine the energy expression by an intrinsic energy in the next subsection. The outcome implies that the total energy can be expressed by the velocity and the momentum as folows, which we will explain in detail later then:

$$
E=\mathbf{v} \cdot \mathbf{p}=v p
$$

Let us define the perpendicular component of a momentum to the distance direction as the "rest-energy momentum" as shown below, where $\theta$ is the angle of the momentum to and $\mathbf{u}_{\mathrm{r}}$ is the unit vector of a direction orthogonal to the distance direction:

$$
\begin{aligned}
& \mathbf{p}_{\mathbf{r}}=p \sin \theta \mathbf{u}_{\mathbf{r}}, \\
& p_{r}=p \sin \theta, \quad \mathbf{u}_{\mathbf{r}} \cdot \mathbf{u}_{\mathbf{r}}=1 .
\end{aligned}
$$

The rest energy is given as follows for the distance direction:

$$
E_{r}=\mathbf{v}_{\mathbf{r}} \cdot \mathbf{p}_{\mathbf{r}}=v p \sin ^{2} \theta=E \sin ^{2} \theta .
$$

- A one-directional force (referred to as the "fundamental force") works between two rest energy momentums as given by the following formula. Here, $d$ is the distance between the two energies, and $K_{f}$ is the "fundamental force constant":

$$
F=K_{f} \frac{\mathbf{p}_{\mathbf{r} \mathbf{1}} \cdot \mathbf{p}_{\mathbf{r} \mathbf{2}}}{d^{2}}=K_{f} \frac{p_{1} p_{2}}{d^{2}} \sin \theta_{1} \sin \theta_{2}=K_{f} \frac{E_{1} E_{2}}{v_{1} v_{2} d^{2}} \sin \theta_{1} \sin \theta_{2} .
$$

If the two energy movements are not in a single plane, let $\theta_{p}$ be the angle between the two planes consisting of the distance direction and the momentum $\mathbf{p}_{\mathbf{1}}$ or $\mathbf{p}_{\mathbf{2}}$. The force is then given as follows (see Fig. 1):

$$
\begin{aligned}
& \mathbf{p}_{\mathbf{1}} \cdot \mathbf{p}_{\mathbf{2}}=p_{1} p_{2} \cos \theta_{p}, \quad \mathbf{p}_{\mathbf{r} \mathbf{1}} \cdot \mathbf{p}_{\mathbf{r} \mathbf{2}}=p_{r 1} p_{r 2} \cos \theta_{p}, \\
& F=K_{f} \frac{\mathbf{p}_{\mathbf{r} \mathbf{1}} \cdot \mathbf{p}_{\mathbf{r} \mathbf{2}}}{d^{2}}=K_{f} \frac{p_{1} p_{2}}{d^{2}} \cos \theta_{p} \sin \theta_{1} \sin \theta_{2} .
\end{aligned}
$$




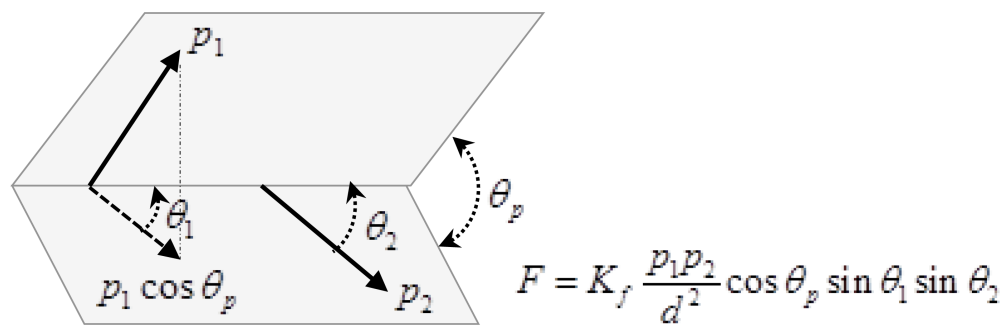

Fig. 1. Fundamental force working on momentums.

- A minus force is attractive and accelerates the two energy pieces to the center of them. It works on the energy movements causing them to bend and make a single circle. A plus force is repulsive and accelerates the pieces outward from the center.

- Take a case of antiparallel movements with velocities $v$ and $-v\left(\theta_{1}=\pi / 2\right.$, $\left.\theta_{2}=-\pi / 2\right)$. The two energy movements that are apart by $d$ make a circulation with the radius $\mu=d / 2$. The force and the distance between the two energies get constant as shown below. Call $v_{c}=\mu \omega$ (equal to $v$ in this case) as the "circulating velocity":

$$
F=K_{f} \frac{p_{1} p_{2}}{d^{2}} \sin \frac{\pi}{2} \sin \frac{-\pi}{2}=-K_{f} \frac{p_{1} p_{2}}{4 \mu^{2}}=-K_{f} \frac{E_{1} E_{2}}{4 v_{c}^{2} \mu^{2}} .
$$

- In the case of an energy circulation, the rest-energy momentum for an orthogonal direction to the circulation plane is zero in total. It is zero for not only orthogonal but also for any direction:

$$
\mathbf{p}_{\mathbf{c r}}=\mathbf{p}_{\mathbf{c}} \sin \theta=\sin \theta \oint p \cos \omega t d \omega t=0 .
$$

On the other hand, the rest energy is the total energy $E_{r}=v_{c} p_{c}$ for any direction. $p_{c}$ is the sum of the magnitudes of momentum for one circle $p_{c}=\oint p d \omega t$.

- The "gravitational force" works between two rest energies as shown below, where $K_{g}$ is the "gravitational force constant by energy" and $m$ is the mass to be discussed in the next subsection:

$$
F=-K_{g} \frac{E_{1} E_{2}}{d^{2}}=-K_{g} v_{c}^{2} \frac{p_{1} p_{2}}{d^{2}}=-K_{g} v_{c}^{4} \frac{m_{1} m_{2}}{d^{2}}=-G \frac{m_{1} m_{2}}{d^{2}} .
$$

According to the energy circulation theory, the intrinsic energy is relative while the total energy is absolute. Take a case that the circulation $\varphi_{x y}$ of an intrinsic energy $E_{0}$ in $X-Y$ shows the total energy $E_{1} . E_{1}$ acts as an intrinsic energy and further circulates in $Y-Z$ by $\varphi_{y z}$, then exhibits total energy of $E_{2}$. We can alternatively perceive $E_{2}$ as that the circulation of $E_{0}$ in $Y-Z$ by $\varphi_{y z}$ acts as an intrinsic energy and it circulates in $X-Y$ by $\varphi_{x y}$. 


\subsection{Energy-mass relation}

As mentioned in Sec. 1, in standard physics, the rest mass is regarded to be invariant by frame selection from the special relativity. However, the rest energy should depend on a measuring frame. Based on the energy circulation theory, we here propose key aspects on the mass including the definition of the term "mass".

There are two ways to express an energy; one is by the total energy with its internal motion and the other is by the intrinsic energy and its moving speed. An example of the first one is an energy distribution expressed by

$$
\mathbf{E}=E \varphi, \quad \boldsymbol{\mu}=\mu \varphi, \quad \varphi=\exp (i \omega t) .
$$

$E$ is the total energy and the wave function $\mu \varphi$ shows an internal circulation at the speed $v_{c}=\mu \omega$. If an intrinsic energy spreads even on the whole circumference, the total energy is also even on the circumference but contains an internal motion expressed by the wave function.

Let us express the same energy by the second-type expression using an intrinsic energy. It is likely that the total energy is proportional to the quantity of the intrinsic energy and the square of the velocity. Let us define the term "mass" as a quantity of an intrinsic energy and the "momentum" as shown below, where $v$ is the moving speed of the intrinsic energy:

$$
m \equiv E / v^{2}, \quad p \equiv m v, \quad E=m v^{2}=p v .
$$

Especially, please note that $E$ is the total energy but not kinetic energy. We can regard that the total energy has the property of momentum. The energy circulation shown by Eq. (2.12) is now expressed by the intrinsic energy as follows:

$$
E=m v_{c}^{2}=m \mu^{2} \omega^{2} .
$$

It can act as the rest energy for any direction whereas the rest-energy momentum is zero in total.

A mass is not proportional to the total energy if the velocity varies. Once a velocity is fixed as constant such as a common circulating velocity, mass and total energy are in proportion. When we express an energy by an intrinsic energy and its velocity like Eq. (2.13), let us refer it to as the "dynamic energy". The mass and the velocity are relative to a frame while the total energy is absolute. Take an energy expressed by Eq. (2.14). By a frame moving at $-v$ linearly, the intrinsic energy $M$ moves at $v$ and shows the following total energy:

$$
E=M v^{2} .
$$

By the stationary frame attached to the subject, the intrinsic energy for $v=0$ is not defined and there exists only the rest energy $E$ for the direction of $v$. For the direction of the circumference of the circulation of the intrinsic energy $m$, the energy $E$ is a dynamic energy shown by Eq. (2.14). There is the following variation in expressing the total energy:

$$
m v_{c}^{2}=E=M v^{2}
$$


Take a frame in which an energy $E_{r}$ is stationary as the initial state. Add an energy $\Delta E$ and accelerate the original energy to move at $v$. The total energy is $E_{t}=E_{r}+\Delta E$. We can express it as a dynamic energy of the intrinsic energy $M_{t}$ moving at $v$ :

$$
E_{t}=M_{t} v^{2}
$$

Let us define the "kinetic energy" as the difference $\Delta E$ from the total energy to the rest energy:

$$
E_{k} \equiv E_{t}-E_{r}
$$

The kinetic energy is equal to the work given from the outside or the change in the potential energy. Refer the circulating intrinsic energy, which provides a rest energy, as the "rest mass":

$$
E_{r}=m_{0} v_{c}^{2}
$$

The total energy $E_{t}$ is then expressed as follows:

$$
E_{t}=m_{0} v_{c}^{2}+E_{k} .
$$

If we take the co-moving frame attached to the subject, the total energy is a rest energy, which can be expressed as a dynamic energy from the circulation of $M_{0}$ at $V_{c}$ :

$$
E_{t}=M_{0} V_{c}^{2} .
$$

Depending on the frame and direction, the expression by an intrinsic energy varies as follows:

$$
E_{t}=M_{t} v^{2}=m_{0} v_{c}^{2}+E_{k}=M_{0} V_{c}^{2} .
$$

$M_{0}$ is the "rest mass by the co-moving frame". It varies by the frame's moving speed $v$. Equation (2.19) is a case for $v=0$ of Eq. (2.21). We propose that $M_{0}$ should act as the working mass of the motion equation $F=M_{0} \alpha$ for the direction of $v$ (if $v=0$, $F=m_{0} \alpha$ ). The rest energy should be the resistance to be accelerated by a force. In fact, however, treating the circulating velocity $v_{c}$ as constant, instead of the rest energy $m_{0} v_{c}^{2}$ we use the intrinsic energy $m_{0}$, which we call as the rest mass. We plan to report on the kinetics of the circulating energy in a medium by a separate paper.

\section{Intra-Circulation and Inter-Circulation Interactions by the Fundamental Force}

\subsection{Intra-circulation force}

Let us examine a force between two local energy parts that are apart by a central angle $\theta$ of an energy circulation $\mathbf{E}=E \varphi, \boldsymbol{\mu}=\mu \varphi, \varphi=\exp (i \omega t)$. The distance between the two parts is

$$
d=2 \mu \sin \frac{\theta}{2}
$$




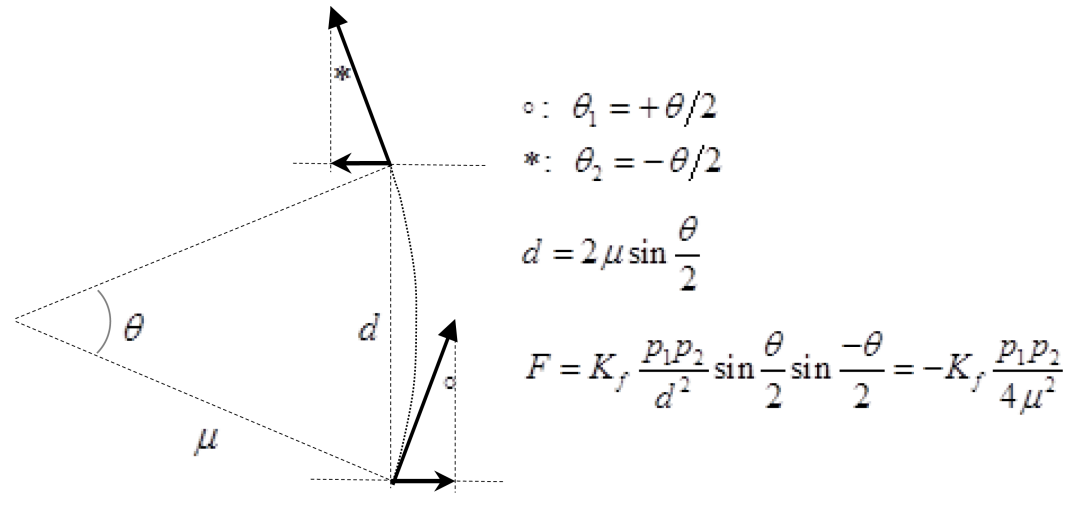

Fig. 2. Intra-circulation force.

The respective angles of the local momentums to the distance direction are $\theta / 2$ and $-\theta / 2$. The force between the two parts is given as follows:

$$
F=K_{f} \frac{\Delta p_{0} \Delta p_{\theta}}{d^{2}} \sin \frac{\theta}{2} \sin \frac{-\theta}{2}=-K_{f} \frac{\Delta p_{0} \Delta p_{\theta}}{d^{2}} \sin ^{2} \frac{\theta}{2} .
$$

Inputing Eq. (3.1), we get the formula of the force as follows, where $v_{c}=\mu \omega$ (see Fig. 2):

$$
F=-K_{f} \frac{\Delta p_{0} \Delta p_{\theta}}{4 \mu^{2}}=-K_{f} \frac{\Delta E_{0} \Delta E_{\theta}}{4 v_{c}^{2} \mu^{2}}
$$

It is independent of the angle $\theta$ or the distance $d$. We get the centripetal and the tangential components of the sum of the forces with the whole circumference for a part as follows:

$$
\begin{gathered}
c F_{\perp}=-K_{f} \sum_{\theta=0}^{2 \pi} \frac{\Delta p_{0} \Delta p_{\theta}}{4 \mu^{2}} \sin \frac{\theta}{2}=-K_{f} \frac{p \Delta p}{4 \mu^{2}} \int_{0}^{2 \pi} \sin \frac{\theta}{2} d \theta=-K_{f} \frac{p \Delta p}{\mu^{2}}=-K_{f} \frac{E \Delta E}{v_{c}^{2} \mu^{2}} \\
c F_{/ /}=-K_{f} \sum_{\theta=0}^{2 \pi} \frac{\Delta p_{0} \Delta p_{\theta}}{4 \mu^{2}} \cos \frac{\theta}{2}=-K_{f} \frac{p \Delta p}{4 \mu^{2}} \int_{0}^{2 \pi} \cos \frac{\theta}{2} d \theta=0 .
\end{gathered}
$$

Any local part of an energy circulation receives a centripetal force shown by Eq. (3.4). The horizontal force is set off and zero.

\subsection{Inter-circulation force}

If we take the whole circulation of energy totally, its rest-energy momentum is zero for any direction as shown by Eq. (2.10). However, between two isolated circulations with a relatively short distance, the force works based on local energy movements. Take a pair of conjugate circulations. There are two kinds of separation of 
circulations; the "orthogonal interaction (separation)", in which the circulations separate in the direction of the common axis, and the "flat interaction (separation)", in which they slide and separate within the common circulation plane. Let us examine the forces between two conjugate circulations of $\omega$ and $-\omega$ that are apart by $d$ for the respective separations.

\subsubsection{Inter-circulation force by the orthogonal interaction}

If the distance $d$ is very small compared with the radius $\mu$ of the circulations, we may approximate the force by Eq. (2.6) with $\theta_{1}=\pi / 2, \theta_{2}=-\pi / 2$. At a specific value of $d$ depending on the momentum, two opposite speed $\pm \mu \omega$ movements in the circumference at a local region make a local circulation in the plane of the tangential direction and the distance $d$ with the same circulating velocity $\mu \omega$. The local circulations lie up and fill up the whole circumference of the original circle. Thus, conjugate circulations $\varphi$ and $\varphi^{*}$ attach to each other with a tiny distance.

When the distance $d$ gets large, the energy movement of the counter-piece expands to the whole cycle. Divide the circulating momentum collectively into two parts; $\mathbf{p}_{0}$ for the arc $-\pi / 2<\theta \leq \pi / 2$ and $\mathbf{p}_{\pi}$ for the arc $\pi / 2<\theta \leq 3 \pi / 2$. The force between orthogonally separated conjugate circulations is given as follows, where $E_{0}$ and $E_{\pi}$ are respective energies for $p_{0}$ and $p_{\pi}$ :

$$
\begin{aligned}
F & =K_{f}\left(\frac{\mathbf{p}_{\mathbf{0}}^{+} \cdot \mathbf{p}_{\mathbf{0}}^{-}+\mathbf{p}_{\pi}^{+} \cdot \mathbf{p}_{\pi}^{-}}{d^{2}}+\frac{\mathbf{p}_{\mathbf{0}}^{+} \cdot \mathbf{p}_{\pi}^{+}+\mathbf{p}_{\pi}^{-} \cdot \mathbf{p}_{\mathbf{0}}^{-}}{d^{2}+4 \mu^{2}}\right) \\
& =-K_{f} \frac{8 p_{0} p_{\pi} \mu^{2}}{d^{4}\left(1+4 \mu^{2} / d^{2}\right)}=-8 \mu^{2} K_{f} \frac{E_{0} E_{\pi}}{v_{c}^{2} d^{4}\left(1+4 \mu^{2} / d^{2}\right)}
\end{aligned}
$$

If $d \gg \mu$, we can approximate it as follows: It is attractive and inversely proportional to the fourth power of the distance:

$$
F \approx-8 \mu^{2} K_{f} \frac{p_{0} p_{\pi}}{d^{4}}=-8 \mu^{2} K_{f} \frac{E_{0} E_{\pi}}{v_{c}^{2} d^{4}} .
$$

\subsubsection{Inter-circulation force by the flat interaction}

The following fundamental force works between the two slid circulations of a conjugate pair in a single plane. The signs of momentums at the neighboring side are same, i. e., $(+-)(-+)$ :

$$
\begin{aligned}
F & =K_{f}\left(\frac{\mathbf{p}_{0}^{+} \cdot \mathbf{p}_{\pi}^{+}}{(d+2 \mu)^{2}}+\frac{\mathbf{p}_{\pi}^{-} \cdot \mathbf{p}_{\mathbf{0}}^{-}}{(d-2 \mu)^{2}}+\frac{\mathbf{p}_{\mathbf{0}}^{+} \cdot \mathbf{p}_{\mathbf{0}}^{-}}{d^{2}}+\frac{\mathbf{p}_{\pi}^{-} \cdot \mathbf{p}_{\pi}^{+}}{d^{2}}\right) \\
& =K_{f} p_{0} p_{\pi}\left(\frac{2 d^{2}+8 \mu^{2}}{\left(d^{2}-4 \mu^{2}\right)^{2}}-\frac{2}{d^{2}}\right) .
\end{aligned}
$$

For $0<d<2 \mu$, the two circulations are not separated enough and the force works on them causing them to return to overlap. For $2 \mu<d \approx 2 \mu$, the strong repulsive 
force works, approximated as

$$
F \approx K_{f} \frac{\mathbf{p}_{\pi}^{-} \cdot \mathbf{p}_{0}^{-}}{(d-2 \mu)^{2}}=K_{f} \frac{p_{0} p_{\pi}}{(d-2 \mu)^{2}}=K_{f} \frac{E_{0} E_{\pi}}{v_{c}^{2}(d-2 \mu)^{2}} .
$$

For $d \gg 2 \mu$, the force can be approximated as follows: It is repulsive for a conjugate pair and inversely proportional to the fourth power of the distance:

$$
F=K_{f} p_{0} p_{\pi} \frac{24 d^{2} \mu^{2}-32 \mu^{4}}{d^{2}\left(d^{2}-4 \mu^{2}\right)^{2}} \approx 24 \mu^{2} K_{f} \frac{p_{0} p_{\pi}}{d^{4}}=24 \mu^{2} K_{f} \frac{E_{0} E_{\pi}}{v_{c}^{2} d^{4}} .
$$

In the case of interactions between same-sign energy circulations (not a conjugate pair), the force by the orthogonal interaction is repulsive, and the force by the flat interaction is attractive.

\subsection{Intra-circulation force between localized intrinsic energies}

Take a case that multiple localized intrinsic energies (massive substances) are circulating at $V_{c}$ on the circumference of a radius $r$. Let $E_{0}$ be the intrinsic energy of a piece and $\Sigma_{0}$ be the sum of the whole intrinsic energies. The piece has the momentum $P_{c}=E_{0} V_{c}$ and the total energy $E_{t}=E_{0} V_{c}^{2}$. The piece receives the following centripetal force by the intra-circulation interaction with the whole circulation based on the momentum:

$$
F=-K_{f} V_{c}^{2} \frac{E_{0} \Sigma_{0}}{r^{2}}
$$

The force works in addition to the gravity. Examples for the force are rings of a planet, stars within a galaxy and binary stars.

In the case that a small intrinsic energy $E_{1}$ circulates at $V_{1}$ around a huge energy $E_{2}$, the momentum of $E_{2}$ is almost zero as it is stationary while $E_{1}$ has the momentum $p_{1}=E_{1} V_{1}$. Accordingly, there hardly works the fundamental force between $E_{1}$ and $E_{2}$. We can approximate that only the gravity works between a planet and the star in a stellar system.

\section{Evolution of the Universe}

\subsection{Initial energy and the first separation}

Provide that the initial energy exhibits vibrations in multiple dimensions. Let $M$ be the number of dimensions. We can express the energy distribution using wave functions by

$$
E \phi_{M}=\frac{E}{2 M} \sum_{k=1}^{M}\left(\varphi_{k}+\varphi_{k}^{*}\right) \mathbf{e}_{\mathbf{k}} \quad\left(\mathbf{e}_{\mathbf{k}}=\text { base vector }\right) .
$$

As discussed in Sec. 2 we can express wave functions by a simple circulation such as Eqs. (2.1) and (2.2). We may express the initial $M$-dimensional energy also as a 
one-dimensional vibration by

$$
E \phi_{1}=\frac{E}{2}\left(\varphi_{k}+\varphi_{k}^{*}\right)
$$

Vibrations in other dimensions are treated as stationary at each center, only contributing to the energy. We can define two ways of expressing energy; one is energy "in motion" and the other is the stationary energy, i.e., "quantized". It is a new definition of "quantization" different from the term in standard physics. However, we guess the new definition could cover the nature of the standard term quantization. In order to exhibit a stationary state, energy should circulate. When an energy movement is quantized in a dimension, which we refer to as the "hidden dimension",

$$
\text { in motion: } \frac{1}{2}\left(\varphi+\varphi^{*}\right)=\cos \omega t \leftrightarrow \text { quantized: } \varphi=\varphi^{*}=1 \text {. }
$$

Take $\omega_{0}$ as the lowest frequency among all the dimensions. The frequencies in other dimensions should be integral multiples of $\omega_{0}$ in order to provide an $M$-dimensional vibration:

$$
\varphi_{k}=\exp \left(i \omega_{k} t\right), \quad \omega_{k}=n_{k} \omega_{0} \quad\left(n_{k}=\text { integer } \geq 1\right) .
$$

There is not an absolute time to express movements. The relativity of a phase $\theta_{k}=\omega_{k} t$ among the dimensions exists. Smaller the time we take, larger the frequency is for the same phase. The vibration showing the longest wave period (lowest frequency) can work as a "tracing dimension", which is defined so that by its value variation it expresses value changes (movements) in other dimensions (the "space") ${ }^{10}$ A tracing dimension works as a "time". The value of time is a single point crossing with a single space location. We will discuss a common time for plural locations later. Define the "original time" $\tau$ as

$$
\theta_{0}=\omega_{0} \tau
$$

by taking $\omega_{0}$ as a constant. $\theta_{0}$ is the varying phase by $\tau$ in the lowest-frequency dimension $X$. Let $\mu_{0}$ be the radius. The energy distribution and the location in $X$ are

$$
\varphi_{x}=\exp \left(i \omega_{0} \tau\right), \quad E \phi_{x}=\frac{E}{2}\left(\varphi_{x}+\varphi_{x}^{*}\right), \quad \mathbf{X}=\mu_{0}\left(\varphi_{x}+\varphi_{x}^{*}\right) .
$$

A tracing dimension is essential in order to express the movement of energy.

If a dimension shows a frequency $N \omega_{0}(N=$ integer $\geq 2)$, we can express its wave function by the combination of frequencies $(N-1) \omega_{0}$ and $\omega_{0}$. When we treat the vibration $\exp \left(i(N-1) \omega_{0} \tau\right)$ as quantized in a steady state, it becomes one and the energy vibrates by $\omega_{0}$. The lowest frequency plays a key role to express an energy movement:

$$
\varphi_{N}=\exp \left(i N \omega_{0} \tau\right)=\exp \left(i(N-1) \omega_{0} \tau\right) \exp \left(i \omega_{0} \tau\right) \leftrightarrow \varphi_{1}=\exp \left(i \omega_{0} \tau\right)
$$


The real nature of a one-dimensional vibration is a pair of conjugate circulations in a two-dimensional plane. Take a pair in $X_{1}$ and $X_{2}$ as shown below:

$$
\begin{aligned}
& \varphi_{12}=\exp \left(i \omega_{0} \tau\right), \quad \mathbf{E}_{2}=\frac{E}{2}\left(\varphi_{12}+\varphi_{12}^{*}\right), \quad \mathbf{X}_{1}=\mu_{0}\left(\varphi_{12}+\varphi_{12}^{*}\right), \\
& \mathbf{X}_{\mathbf{2}}=-i \mu_{0}\left(\varphi_{12}+\varphi_{12}^{*}\right) .
\end{aligned}
$$

Take the third and fourth directions $X_{3}$ and $X_{4}$ orthogonal to each other and also to $X_{1}$ and $X_{2}$. The energy circulates in $X_{3}$ and $X_{4}$ similarly as follows:

$$
\begin{aligned}
& \varphi_{34}=\exp \left(i \omega_{0} \tau\right), \quad \mathbf{E}_{\mathbf{2}}=\frac{E}{2}\left(\varphi_{34}+\varphi_{34}^{*}\right), \quad \mathbf{X}_{\mathbf{3}}=\mu_{0}\left(\varphi_{34}+\varphi_{34}^{*}\right), \\
& \mathbf{X}_{4}=-i \mu_{0}\left(\varphi_{34}+\varphi_{34}^{*}\right) .
\end{aligned}
$$

We can express the two circulations by a four-dimensional expression $\mathbf{E}_{4}$ as shown below:

$$
\mathbf{E}_{4}=\frac{E}{4}\left(\varphi_{12}+\varphi_{12}^{*}\right)\left(\varphi_{34}+\varphi_{34}^{*}\right)
$$

Simultaneous separations of the two pairs of circulations are possible; one $\left(X_{1}-X_{2}\right)$ is orthogonal and the other $\left(X_{3}-X_{4}\right)$ is flat:

$$
\frac{E}{4}\left(\varphi_{12}+\varphi_{12}^{*}\right)\left(\varphi_{34}+\varphi_{34}^{*}\right) \rightarrow \frac{E}{2} \varphi_{12} \varphi_{34}+\frac{E}{2} \varphi_{12}^{*} \varphi_{34}^{*}
$$

Only just after the flat separation of $\varphi_{34}$ and $\varphi_{34}^{*}$ during $2 \mu<r \approx 2 \mu$, a strong repulsive force shown by Eq. (3.10) works between the energy pieces, but immediately fades out as shown by Eq. (3.11). Let us call each piece of the energy as a "universe". Name the separation as the "Giant Separation". A universe has the opposite-sign angular momentums in $X_{1}-X_{2}$ and $X_{3}-X_{4}$ to those of the other universe. The other universe should have an opposite chirality of energy movement to that of our universe due to the different signs of the spin quantum numbers. The simultaneous occurrence of the orthogonal and the flat separations brings some asymmetry in a four-dimensional space; this is the reason why we use a fourdimensional expression.

\subsection{Expansion of the $4 D$ spherical universe}

Newly take the frame attached to our universe. The energy distribution of the universe after the Giant Separation expressed by $\varphi_{12}, \varphi_{34}$ is in a $3 \mathrm{D}$ surface of a $4 \mathrm{D}$ sphere. Let us express the $4 \mathrm{D}$ sphere by $4 \mathrm{D}$ polar coordinates:

$$
\mathbf{X}=\left(\mu_{0} \theta_{1} \theta_{2} \theta_{3}\right)=\left(\mu_{0} \boldsymbol{\theta}\right) .
$$

By Cartesian coordinates it is shown as follows, where $i, j, k$ denote quaternion (imaginary) units corresponding to base vectors for $X_{2}, X_{3}$ and $X_{4}$, respectively:

$$
\begin{aligned}
\mathbf{X} & =X_{1}+i X_{2}+j X_{3}+k X_{4} \\
& =\mu_{0}\left(\cos \theta_{1}+i \sin \theta_{1} \cos \theta_{2}+j \sin \theta_{1} \sin \theta_{2} \cos \theta_{3}+k \sin \theta_{1} \sin \theta_{2} \sin \theta_{3}\right) .
\end{aligned}
$$


Input the circulations $\varphi_{12}, \varphi_{34}$, then we get the following function for the energy movement:

$$
\mathbf{X}=\mu_{0}\left(\cos \omega_{0} \tau+i \sin \omega_{0} \tau \cos \theta_{2}+j \sin \omega_{0} \tau \sin \theta_{2} \cos \omega_{0} \tau+k \sin \omega_{0} \tau \sin \theta_{2} \sin \omega_{0} \tau\right)
$$

For a circulation in $X_{1}-X_{2}$, take the respective base vectors as follows:

$$
\mathbf{e}_{\mathbf{0}} \equiv \cos \theta_{1}+i \sin \theta_{1}, \quad \mathbf{e}_{1} \equiv \cos \left(\theta_{1}+\pi / 2\right)+i \sin \left(\theta_{1}+\pi / 2\right)=-\sin \theta_{1}+i \cos \theta_{1} .
$$

At any angle $\theta_{1}, \mathbf{e}_{\mathbf{0}}$ is the radial direction and $\mathbf{e}_{\mathbf{1}}$ is the tangential one. A value in $\mathbf{e}_{\mathbf{1}}$ shows an arc distance on the circumference. The energy circulation $\varphi_{12}$ with the radius $\mu_{0}$ is expressed by $\mathbf{e}_{\mathbf{0}}$ and $\mathbf{e}_{1}$ as shown below where $v_{c}=\mu_{0} \omega_{0}$ is the circulating velocity:

$$
\begin{aligned}
& \text { Radius: } \mu_{0} \varphi_{12}=\mu_{0}\left(\cos \omega_{0} \tau+i \sin \omega_{0} \tau\right)=\mu_{0} \mathbf{e}_{\mathbf{0}}, \\
& \text { Arc: } v_{c} \tau \mathbf{e}_{\mathbf{1}}=\mu_{0} \omega_{0} \tau \mathbf{e}_{\mathbf{1}}, \\
& \text { Collective: } \mu_{0} \mathbf{e}_{\mathbf{0}}+v_{c} \tau \mathbf{e}_{\mathbf{1}}=\mu_{0}\left(\mathbf{e}_{\mathbf{0}}+\omega_{0} \tau \mathbf{e}_{\mathbf{1}}\right)
\end{aligned}
$$

In fact, the two-dimensional energy distribution in the plane $j-k$ circulates on the arc $\mathbf{e}_{\mathbf{1}}$ as shown by Eq. (4.9). The directions $j$ and $k$ are orthogonal to the plane $\mathbf{e}_{\mathbf{0}}-\mathbf{e}_{\mathbf{1}}$. Define the following $3 \mathrm{D}$ space vector:

$$
\mathbf{R} \equiv i \cos \theta_{2}+j \sin \theta_{2} \cos \omega_{0} \tau+k \sin \theta_{2} \sin \omega_{0} \tau
$$

We can rewrite the energy distribution shown by Eq. (4.9) as follows:

$$
\begin{gathered}
\mathbf{X}=\mu_{0}\left(\cos \omega_{0} \tau+\mathbf{R} \sin \omega_{0} \tau\right) \\
\mathbf{X}=\mu_{0}\left(\mathbf{e}_{\mathbf{0}}+\omega_{0} \tau \mathbf{e}_{\mathbf{1}} \cos \theta_{2}+\sin \theta_{2}\left(j \cos \omega_{0} \tau+k \sin \omega_{0} \tau\right)\right) \equiv \mu_{0}\left(\mathbf{e}_{\mathbf{0}}+\mathbf{r}\right) .
\end{gathered}
$$

Equation (4.13) shows that the $3 \mathrm{D}$ energy distribution $\mu_{0} \mathbf{R}$ circulates in $X_{1}-X_{2}$ by $\mu_{0} \varphi_{12}$ in the $4 \mathrm{D}$ space. We can also express the same energy distribution by the radius $\mu_{0} \mathbf{e}_{\mathbf{0}}$ and the $3 \mathrm{D}$ surface space $\mu_{0} \mathbf{r}$ as shown by Eq. (4.14). $\mathbf{e}_{\mathbf{1}}$ is an arc in the $4 \mathrm{D}$ space but can be treated as linear in the $3 \mathrm{D}$ surface space $\mu_{0} \mathbf{r}$, where $\mathbf{e}_{\mathbf{1}}, j, k$ form $3 \mathrm{D}$ Cartesian coordinates. In the $3 \mathrm{D}$ world, energy moves at a velocity $\mu_{0} \omega_{0} \cos \theta_{2}$ varying by $\theta_{2}$ in the common space direction $\mathbf{e}_{1}$. Refer the circulation $\mu_{0} \varphi_{12}$ to as the "radius circulation".

The energy circulation $\mu_{0} \varphi_{34}$ in $X_{3}-X_{4}$ becomes the second term of the following equation in the $3 \mathrm{D}$ surface space:

$$
\mu_{0} \mathbf{r}=\mu_{0} \omega_{0} \tau \mathbf{e}_{1} \cos \theta_{2}+\mu_{0} \sin \theta_{2}\left(j \cos \omega_{0} \tau+k \sin \omega_{0} \tau\right)
$$

This is a circulation in two space dimensions $j$ and $k$. However, the radius varies by the angle $\theta_{2}$, which is a parameter to express the location and spreads in $0 \leq \theta_{2} \leq \pi$. The energy is filled up in the $3 \mathrm{D}$ space $\mu_{0} \mathbf{r}$ without a bulk. Therefore, this is a rotation rather than a circulation. Let us refer it to as the "space rotation". 
The energy of the universe rotates around an axis and moves linearly in the axis direction. The axis is a circle in the $4 \mathrm{D}$ space.

In order to show a steady-state circulation, the centripetal force should balance with the centrifugal force. As mentioned in Sec. 3.2, a pair of conjugate circulations makes local circulations with the distance direction, which lie up and fill up the whole circumference of the original circle. Therefore, the centrifugal force of the pair is not only in the plane of the circulation but also in the distance direction. Once the pair is separated, the centrifugal force is only in the circulation plane. After the Giant Separation, the circulating velocity became too fast to keep $\mu_{0}$ and $\omega_{0}$ in each circulation. Then the circulations turned to expand (the "Big Bang") and are now still on the way, not yet reached the new balance. We can express the cosmological expansion by that of the radius circulation $\mu_{0} \varphi_{12}$. We may treat the expansion as the sum of all local energy separations to opposite directions. The initial speed of the expansion in the radius was $v_{0}=\mu_{0} \omega_{0}$ equal to the original circulating velocity $v_{c}$. The radial expansion speed decelerates by the intra-circulation force shown by Eq. (3.4) based on the radius circulation. For a given minute central-angle area $\Delta \boldsymbol{\theta}=\left(\Delta \theta_{1} \Delta \theta_{2} \Delta \theta_{3}\right)$ the energy $\Delta E_{0}$ is invariant by time, and shows a onedimensional separation with the opposite area energy $\Delta E_{\pi}$ at $\Delta \boldsymbol{\theta}+\pi$. The separation occurs simultaneously at all regions of the circumference. Let $x$ be the radius. The initial circulation $\mu_{0} \varphi_{12}$ expands with the new radius $x$ and frequency $\omega$, which vary by time:

$$
\mathbf{X}=x(\sin \omega \tau+i \cos \omega \tau) .
$$

The centripetal force that a minute area energy $\Delta E$ receives from the whole energy $E_{U}$ of the circulation (universe) is given as follows from Eq. (3.4), where $v_{c}=x \omega$ :

$$
F=-K_{f} \frac{E_{U} \Delta E}{v_{c}^{2} x^{2}} .
$$

From the conservation of the sum of the potential and the kinetic energies, we get the expansion speed of the radius as follows:

$$
\begin{gathered}
E_{p}(x)-E_{p}\left(x_{0}\right)+E_{k}(x)=E_{k}\left(x_{0}\right), \\
E_{k}\left(x_{0}\right)=\int_{x_{0}}^{x} K_{f} \frac{E_{U} E\left(x_{0}\right)}{v_{o}^{2} x^{2}} d x+E_{k}(x)=K_{f} \frac{E_{U} E\left(x_{0}\right)}{v_{o}^{2}}\left(\frac{1}{x_{0}}-\frac{1}{x}\right)+E_{k}(x), \\
E\left(x_{0}\right)=m v_{c}^{2}=m v_{0}^{2}, \quad E_{k}\left(x_{0}\right)=\frac{1}{2} m v_{0}^{2}, \quad E_{k}(x)=\frac{1}{2} m v^{2}, \\
v_{0}^{2}=2 K_{f} E_{U}\left(\frac{1}{x_{0}}-\frac{1}{x}\right)+v^{2} \\
x_{0}, v_{0}: \text { initial values, } m: \text { mass (intrinsic energy), } \\
v^{2}=2 K_{f} E_{U}\left(\frac{1}{x}-K\right), \quad K \equiv \frac{1}{x_{0}}-\frac{v_{0}^{2}}{2 K_{f} E_{U}},
\end{gathered}
$$




$$
v=\frac{d x}{d \tau}= \pm \sqrt{2 K_{f} E_{U}\left(\frac{1}{x}-K\right)} .
$$

If we use the "Cosmic Unit" for $x$ as one for the maximum radius of the universe, the space expansion speed by $\tau$ is zero at $x=1$, i.e., $K=1$ :

$$
\frac{d x}{d \tau}= \pm \sqrt{2 K_{f} E_{U}\left(\frac{1}{x}-1\right)} .
$$

The "imaginary order of freedom" is defined as follows: If a movement shows a single moving value in one direction (increase or decrease) when traced by another movement, it has the imaginary order of freedom. ${ }^{10}$ In the range $\mu_{0} \leq x<1, x$ shows the imaginary order of freedom when traced by $\tau$. Therefore $x$ can be another tracing dimension. Refer the value of $x$ to as the "Observed Time" $T$. The time intervals $d \tau$ and $d T=d x$ have the relation shown by Eq. (4.22). For our observation time scale, we can regard that the expansion speed is constant and the Observed Time is proportional to the original time:

$$
\frac{d T}{d \tau}=\frac{d x}{d \tau} \approx K_{T}
$$

Let us divide the energy of the universe into two parts; the rest energy and the kinetic energy. The rest energy spreads homogeneously in the space. Refer it to as the "space energy", which corresponds to the vacuum space. In a local space there are two linear energy movements orthogonal to each other derived from the radius circulation and the space rotation. One is in the common direction $\mathbf{e}_{\mathbf{1}}$ at a velocity $x \omega \cos \theta_{2}$, and the other is at a speed $x \omega \sin \theta_{2}$ in a direction depending on $\theta_{3}$ as shown below. $\theta_{2}$ and $\theta_{3}$ are the parameters to show the location $\left(\theta_{3}=\omega \tau\right.$ for energy movement):

$$
x \mathbf{r}=x \omega \tau \mathbf{e}_{1} \cos \theta_{2}+x \sin \theta_{2}\left(j \cos \theta_{3}+k \sin \theta_{3}\right) .
$$

Refer the kinetic energy to as the "apparent energy". The apparent energy is what we detect as energy in the $3 \mathrm{D}$ space. If we take a single intrinsic energy $m$ for the whole apparent energy in a small local space, the energy is shown as follows:

$$
E_{a}=m\left(\left(x \omega \cos \theta_{2}\right)^{2}+\left(x \omega \sin \theta_{2}\right)^{2}\right)=m x^{2} \omega^{2} .
$$

We can also regard it as two dynamic energies by separate intrinsic energies, which are moving in respective directions $\mathbf{e}_{1}$ and $j \cos \theta_{3}+k \sin \theta_{3}$ :

$$
E_{a}=\left(m \cos ^{2} \theta_{2}\right) x^{2} \omega^{2}+\left(m \sin ^{2} \theta_{2}\right) x^{2} \omega^{2} .
$$

This geometry of the energy distribution of the universe is generally called a $3 \mathrm{D}$ sphere. However, let us call the universe as the " $4 \mathrm{D}$ spherical universe" including both the one-dimensional time and the three-dimensional spaces. In order to avoid a confusion, allow to use "3D surface" instead of "3D sphere" for the space. 


\section{Space and Time}

So far we have discussed many important aspects of the time and space. Let us recall and summarize the key features.

\subsection{Space}

When traced by a movement (time), the dimensions in which energy distributions are quantized in a steady state are the hidden dimensions. The other dimensions, in which energy locations are in motion, are the space dimensions. A movement of energy, which shows the imaginary order of freedom, can work as another tracing movement to express value variations in space dimensions.

\subsection{Time}

The magnitude of the radius of the $4 \mathrm{D}$ sphere (Observed Time) can be a tracing dimension common for any locations and any movements in the $3 \mathrm{D}$ space. However, we do not realize it. If we share a common movement, which moves in a constant speed when traced by the Observed Time, it can work as an indirect tracing movement to trace movements at distant locations ("remote tracing") jointly with recorded past information ("chronological recording"). The movement is the "clock", which can work as a time. Let us summarize below the relations between the three kinds of time we introduced so far.

(i) Original time $\tau: \omega_{0} \tau$ (phase in lowest-frequency vibration of the initial energy),

(ii) Observed time $T: \frac{d T}{d \tau}=\frac{d x}{d \tau}=\sqrt{2 K_{f} E_{U}\left(\frac{1}{x}-1\right)} \approx K_{T}$,

(iii) Clock time $t_{c}: \frac{d t_{c}}{d \tau}=\frac{d t_{c}}{d T} \frac{d T}{d \tau} \approx V_{c} K_{T} \quad\left(V_{c} \equiv \frac{d t_{c}}{d T}\right)$.

In order to work as a time two aspects arise; whether direct tracing is possible and whether the base movement is inertial. For setting up a time, we need its base movement, to which we put a certain value as its constant speed, then the time is defined. The base movements for respective times and whether or not they are expected theoretically to be inertial are as follows:

$\tau$ : circumference $\left(\mu_{0} \omega_{0} \tau\right)$ inertial,

$T: 4$-D radius $(\boldsymbol{x}) \quad$ not inertial (inertial for our time scale),

$t_{c}$ : movement of a clock inertial.

"Direct tracing" expresses a single location common in time and in space. The time axis is orthogonal to all space directions. Therefore what traces others should be a different dimension. Only $T$ can be a tracing dimension. The other $\tau$ and $t_{c}$ are a tracing movement in space dimensions. The magnitude of the Observed Time $T$ is the only tracing dimension capable to commonly trace any locations in the 3D space, and accordingly capable to directly trace a movement in the space. 
(a) For actual measurement by two different time points: Possible time interval for actual measurement is too small to detect an acceleration of the $4 \mathrm{D}$ radius $x$. We can treat all types of time as proportional to each other. We do not have to care which type of time we are using but it is enough to set up a standard movement, to which we fix its speed as a constant. Currently the atomic clock of cesium is the standard clock. Its base movement is the propagation of a specific radiation from the atom. The speed set up as a constant is the light speed. When we use $t$ for time afterwards, any type of time is OK unless otherwise refined.

(b) For cosmological observation: We cannot practically detect a very distant object using an unchanging common clock at both locations. The only potential movement that connects to all places of the space is the Observed Time (radius). We detect the present light emitted from an object in the past. During light traveling, the universe has expanded, the light has been redshifted and the light speed has been varying due to the decreasing density of the space energy. As we formerly reported, the Observed Time is suitable to handle these parameters. It can express the ratio of the space expansion, redshift and light speed by simple formulas independently of the expansion speed of the universe. ${ }^{8}$ The Hubble diagram expected from the model using the Observed Time gave an excellent fit to the plots of the observed supernovae. $^{8}$

On the other hand, in order to describe the expansion speed of the universe at a fixed location without movement, the original time is useful. By the Observed Time, the expansion speed of the radius is constant.

\section{Excitation of a Unit Space and Generation of Light}

\subsection{Local circulation of the space energy}

Take Cartesian coordinates attached to a single location in the space from now on. Any location of the 3D space shares an energy distribution with the hidden dimensions. In a vacuum area of the $3 \mathrm{D}$ space, take a very small $3 \mathrm{D}$ ball area of radius $\mu_{0}$, which is the largest radius of the hidden dimensions. Refer the ball area to as the "unit space". Let us express the space energy $E_{\mu}$ in a unit space as a pair of conjugate circulations with the radius $\mu_{0}$ in a space direction $(X)$ and the hidden dimension of the lowest frequency $\omega_{0}$ (referred to as the " $H$-dimension") as below. The real part is for $X$ and the imaginary part is for $H$. It is a one-space-dimensional expression:

$$
\begin{aligned}
E_{\mu} \phi_{1} & =\frac{E_{\mu}}{2}\left(\varphi_{x}+\varphi_{x}^{*}\right), \quad \mathbf{X}=\mu_{0}\left(\varphi_{x}+\varphi_{x}^{*}\right), \\
\mathbf{H} & =-i \mu_{0}\left(\varphi_{x}+\varphi_{x}^{*}\right), \quad \varphi_{x}=\exp \left(i \omega_{0} t\right) .
\end{aligned}
$$

First, we examine a force working in a single circulation $\varphi_{x}$ of the pair. The total energy of the circulation is $E_{\mu} / 2$. The intra-circulation force works between any pieces of energy. An energy piece $\Delta E$ at a minute location receives the forces with energy pieces at all the locations on the whole circumference. The sum of the forces 
results in a one-directional centripetal force shown below from Eq. (3.4):

$$
F=-K_{f} \frac{\left(E_{\mu} / 2\right) \Delta E}{v_{c}^{2} \mu_{0}^{2}}=-K_{f} \frac{E_{\mu} \Delta E}{2 v_{c}^{2} \mu_{0}^{2}} .
$$

Because all forces at any locations are centripetal, we may treat the whole feature of forces on the whole circumference as a two-dimensional composition of one-directional forces between two locations that are apart by an angle $\pi$. Divide the whole energy into two parts; the upper arc $E_{0}$ for $-\pi / 2<\theta \leq \pi / 2$ and the lower arc $E_{\pi}$ for $\pi / 2<\theta \leq 3 \pi / 2, E_{0}=E_{\pi}=E_{\mu} / 4$, where locations are expressed by the angle $\theta$ as follows:

$$
\boldsymbol{\mu}(\theta)=X(\theta)+i H(\theta)=\mu_{0} \exp (i \theta), \quad X(\theta)=\mu_{0} \cos \theta, \quad H(\theta)=\mu_{0} \sin \theta .
$$

The sum of the magnitudes of the centripetal forces is equal to that of the force between localized two energy pieces of $E_{0}$ and $E_{\pi}$ as shown below:

$$
F=-\int_{-\pi / 2}^{\pi / 2} K_{f} \frac{\Delta E(\theta) \Delta E(\theta+\pi)}{v_{c}^{2}\left(2 \mu_{0}\right)^{2}} d \theta=-K_{f} \frac{E_{0} E_{\pi}}{v_{c}^{2}\left(2 \mu_{0}\right)^{2}} .
$$

If we regard the circumference as one direction, the total momentum of the energy circulation is as follows from the energy-mass equation (2.14):

$$
\frac{E_{\mu}}{2}=p_{c} v_{c}, \quad v_{c}=\mu_{0} \omega_{0} .
$$

Define the respective momentums for $E_{0}$ and $E_{\pi}$ :

$$
p_{0} \equiv E_{0} / v_{c}, \quad p_{\pi} \equiv E_{\pi} / v_{c}, \quad p_{0}=p_{\pi}=E_{\mu} / 4 v_{c} .
$$

Give them respective directions by Cartesian complex coordinates of $X$ and $H$. The direction of $\mathbf{p}_{\mathbf{0}}$ is $+i$ and that of $\mathbf{p}_{\pi}$ is $-i$ in accordance with the momentums at $\theta=0$ and $\theta=\pi$ :

$$
\begin{aligned}
& \mathbf{p}_{\mathbf{0}}=i p_{0}, \quad \mathbf{p}_{\pi}=-i p_{\pi}, \\
& \mathbf{p}_{\mathbf{0}} \cdot \mathbf{p}_{\pi}=i p_{0}\left(-i p_{\pi}\right)=p_{0} p_{\pi} .
\end{aligned}
$$

The total centripetal force shown by Eq. (6.2) can be expressed by momentums as follows:

$$
F=-K_{f} \frac{E_{0} E_{\pi}}{v_{c}^{2}\left(2 \mu_{0}\right)^{2}}=-K_{f} \frac{p_{0} p_{\pi}}{\left(2 \mu_{0}\right)^{2}}=-K_{f} \frac{\mathbf{p}_{0} \cdot \mathbf{p}_{\pi}}{\left(2 \mu_{0}\right)^{2}}
$$

We can express the momentums in the hidden dimension $H$ shown as Eqs. (6.5) by a charge. Let us define the "electric charge" as follows:

$$
\begin{gathered}
\mathbf{e} \equiv i \mathbf{p} ; \quad \mathbf{e}_{\mathbf{0}}=i \mathbf{p}_{\mathbf{0}}, \quad \mathbf{e}_{\pi}=i \mathbf{p}_{\pi}, \\
\mathbf{e}_{\mathbf{0}}=-p_{0}=-\frac{E_{\mu}}{4 v_{c}}, \quad \mathbf{e}_{\pi}=p_{\pi}=+\frac{E_{\mu}}{4 v_{c}} .
\end{gathered}
$$


The charge $\mathbf{e}_{\mathbf{0}}$ is negative, and $\mathbf{e}_{\pi}$ is positive from the definitions. We get the following formula by the electric charge for the force:

$$
F=K_{f} \frac{\mathbf{e}_{\mathbf{0}} \cdot \mathbf{e}_{\pi}}{\left(2 \mu_{0}\right)^{2}}
$$

The force is attractive when the two charges have different signs and repulsive for the same sign. By the original energy circulation at $\omega_{0}$, a pair of energy pieces move from $\boldsymbol{\mu}(0)$ to $\boldsymbol{\mu}(\pi)$ and from $\boldsymbol{\mu}(\pi)$ to $\boldsymbol{\mu}(0)$, respectively, but the signs of the charge (momentum) remain invariant at the respective locations. Let us call it as the "electric charge polarization". Define the force expressed by the electric charge as the "electric force". The $X-H$ circulation can move to the next unit spaces, which gives different features of the electric force from the force in an energy circulation in two space dimensions. We will discuss this point later in Sec. 8. The electric charge is only for a momentum in a hidden dimension.

The momentum is a one-dimensional charge, in a direction orthogonal to which the fundamental force works. We especially call the momentum in the hidden dimension of an energy movement in one hidden and one space dimensions as the electric charge. The gravitational force works on a zero-dimensional charge that is the energy. Since the total movement of the apparent energy is in four dimensions, there would exist higher-dimensional forces by a two-dimensional charge and by a three-dimensional charge, respectively.

Now go back to the pair of conjugate circulations $\varphi_{x}$ and $\varphi_{x}^{*}$ in $X$ and $H$. The electric charge polarizations are set off at each other and become zero:

$$
\mathbf{e}_{\mathbf{0}}+\mathbf{e}_{\mathbf{0}}^{*}=i \mathbf{p}_{\mathbf{0}}+i\left(-\mathbf{p}_{\mathbf{0}}\right)=0, \quad \mathbf{e}_{\pi}+\mathbf{e}_{\pi}^{*}=i \mathbf{p}_{\pi}+i\left(-\mathbf{p}_{\pi}\right)=0 .
$$

The energy distribution is

$$
\frac{E_{\mu}}{2}\left(\varphi_{x}+\varphi_{x}^{*}\right)=E_{\mu} \cos \omega_{0} t
$$

The conjugate pair exhibits a one-dimensional energy vibration in the $3 \mathrm{D}$ space. Let us refer the space energy in a unit space to as "spacia", the energy of which is $E_{\mu}$. It constitutes the space energy, which represents the vacuum space of the universe. The direction $X$ discussed above is applicable for any direction in the three space dimensions. The spacia and collectively the space energy appear symmetric and flat in the $3 \mathrm{D}$ space, where energy vibrations in any directions are quantized.

\subsection{Additional energy to the space energy}

The apparent energy is the resource to render an additional energy to a spacia. An apparent energy in a local space has a linear momentum in a space direction. Add $\Delta E_{\mu}$ and excite a spacia:

$$
E_{\mu}^{\#}=E_{\mu}+\Delta E_{\mu}
$$


$\Delta E_{\mu}$ accelerates the circulating velocity of the energy on the circumference. As a potential excitation, let us examine an acceleration of the circulation in the $X-H$ plane. The hidden dimension $H$ should be kept quantized after the excitation. Therefore, the frequency $\omega^{\#}=\omega_{0}+\Delta \omega$ after excitation should be integral $(\geq 2)$ multiple of that at the ground state $\omega_{0}$. Accordingly, we get the relation $\Delta \omega=n \omega_{0}$ $(n=$ integer $\geq 1)$. On the other hand, the radius $\mu_{0}$ remains unchanged because the $H$-dimension is quantized for circulations with any space directions. Consider the excitation by $\Delta E_{\mu}$, which comprises two orthogonal movements, in both conjugate circulations as shown below:

$$
\begin{aligned}
\phi^{\#} & =\frac{1}{2}\left(\varphi^{\#}+\left(\varphi^{*}\right)^{\#}\right)=\frac{1}{2}\left(\exp \left(i\left(\omega_{0}+\Delta \omega\right) t\right)+\exp \left(-i\left(\omega_{0}+\Delta \omega\right) t\right)\right) \\
& =\frac{1}{2}\left(\exp \left(i \omega_{0} t\right) \exp (i \Delta \omega t)+\exp \left(-i \omega_{0} t\right) \exp (-i \Delta \omega t)\right) \\
& =\frac{1}{2}\left(\varphi \exp (i \Delta \omega t)+\varphi^{*} \exp (-i \Delta \omega t)\right) \equiv \frac{1}{2}\left(\varphi \varphi_{\Delta}+\varphi^{*} \varphi_{\Delta}^{*}\right) .
\end{aligned}
$$

If we treat the ground state spacia as a medium for the excitation, the function of the medium shall be regarded as $\varphi=\varphi^{*}=1$. The vibration of the medium for the excitation is given by

$$
\phi_{m}^{\#}=\frac{1}{2}\left(\varphi_{\Delta}+\varphi_{\Delta}^{*}\right)
$$

This signifies the existence of a quantum particle of energy $\Delta E_{\mu}$ in the medium. The frequency of the resulted particle is $\Delta \omega=n \omega_{0}(n=$ integer $\geq 1)$. The energy $\Delta E_{\mu}$ is given as follows:

$$
\begin{aligned}
& E_{\mu}=m_{\mu} \mu_{0}^{2} \omega_{0}^{2}, \\
& \Delta E_{\mu}=m_{\mu} \mu_{0}^{2} \Delta \omega^{2}=n^{2} E_{\mu} .
\end{aligned}
$$

Let us refer the resulted particle in the space energy to as "spacino" ( $S_{1}$ for this case). Rephrase parameters as $\phi_{s} \equiv \phi_{m}^{\#}, \varphi_{x} \equiv \varphi_{\Delta}, \varphi_{x}^{*} \equiv \varphi_{\Delta}^{*}, E_{s} \equiv \Delta E_{\mu}$. The wave function of the spacino and key parameters are as follows:

$$
\varphi_{x}=\exp \left(i n \omega_{0} t\right), \quad E_{s} \phi_{s}=\frac{E_{s}}{2}\left(\varphi_{x}+\varphi_{x}^{*}\right)=E_{s} \cos n \omega_{0} t \quad(n=\text { integer } \geq 1),
$$

Energy: $E_{s}=n^{2} E_{\mu}, \quad$ Radius: $\mu_{s}=\mu_{0}$,

Electric charge: 0, Spin: 0.

The above-mentioned spacino referred to as $S_{1}$ is a pair of conjugate circulations quantized in one space and one hidden dimensions. The quantization in two space dimensions should be also possible. Even its single circulation has no electric charge polarization. Let us call the conjugate pair of it as the $S_{0}$ spacino. Further excitation of the $S_{1}$ spacino could result in quantization in one hidden and two or three space 
dimensions. We may refine the definition of the spacino as a quantized particle in space, whose energy circulations are kept as a conjugate pair. There is no electric charge or circulating momentum in the spacino. The spacino receives the gravitational force and gathers with each other or with other particles. Unless otherwise specified, the term "spacino" shall means the $S_{1}$ spacino of $n=1$.

\subsection{Generation of light}

The spacino $\left(S_{1}, n=1\right)$ has the following conjugate circulations in the hidden $H$ and space $(X)$ dimensions:

$$
E_{s} \phi_{s}=\frac{E_{s}}{2}\left(\varphi_{x}+\varphi_{x}^{*}\right), \quad \varphi_{x}=\exp \left(i \omega_{0} t\right), \quad \mathbf{X}=\mu_{0}\left(\varphi_{x}+\varphi_{x}^{*}\right), \quad \mathbf{H}=-i \mu_{0}\left(\varphi_{x}+\varphi_{x}^{*}\right)
$$

Let us take a single circulation $\varphi_{x}$ or $\varphi_{x}^{*}$, which we refer to as the "photino". Not only a spacino but various quantum particles would have an internal component of a photino. By adding energy $\Delta E$, rotate a photino around a space axis orthogonal to $X$ at the zero point of $X$ by a frequency $\omega$. Let $Z$ be the axis and $Y$ be the space direction orthogonal to $X$ in the rotating plane. The rotation around $Z$ rotates also in the $H-X$ plane other than the $X-Y$ plane. This is a rotation in a four-dimensional space, where a single axis has two rotating planes orthogonal to each other. We can express the rotation by the following function, where $j$ is another imaginary unit orthogonal to $i$ :

$$
\begin{gathered}
\varphi_{x y h}=\cos \omega t+j \sin \omega t+i \sin \omega t \\
\mathbf{X}=\mu_{0} \varphi_{x y h}, \quad \mathbf{Y}=-j \mu_{0} \varphi_{x y h}=\mu_{0}(\sin \omega t-j \cos \omega t) \\
\mathbf{H}=-i \mu_{0} \varphi_{x y h}=\mu_{0}(\sin \omega t-i \cos \omega t)
\end{gathered}
$$

Divide the total energy into the rest energy $E_{s} / 2$ of the photino and the kinetic energy $\Delta E$ by the rotation. When we express the kinetic energy as a dynamic energy of the circulation on the circumference at a speed $v_{c}=\mu_{0} \omega$, the intrinsic energy is not the whole energy of the photino because the photino is rotating at the center. It is equal to that for the internal circulation in $H-X$ of the photino. Let $m_{p}$ be the intrinsic energy. The energy of the photino is given by the intrinsic energy and the circulating velocity as $E_{s} / 2=m_{p} c_{p}^{2}$. The circulating velocity in $H-X$ of the photino is $c_{p}=\mu_{0} \omega_{0}$. Accordingly, the intrinsic energy is

$$
m_{p}=\frac{E_{s}}{2 c_{p}^{2}}=\frac{E_{s}}{2 \mu_{0}^{2} \omega_{0}^{2}} .
$$

We get the following formula of dynamic energy as the kinetic energy by rotation:

$$
\Delta E=m_{p} v_{c}^{2}=\frac{E_{s}}{2 \mu_{0}^{2} \omega_{0}^{2}} \mu_{0}^{2} \omega^{2}=\frac{E_{s}}{2 \omega_{0}^{2}} \omega^{2} .
$$


The kinetic energy has the wave function of Eq. (6.15) and vibrations shown by Eq. (6.16).

Since the kinetic energy by rotation is not quantized, it propagates to surrounding spacias while the photino stops its rotation and stays at the original position. The $H-X$ circulation breaks and the energy moves in $X$. However, the movement keeps the vibration by $\omega$ in $H$ because the hidden $H$-dimension has only the length $2 \mu_{0}$ in any locations of the space. The energy release from the photino is possible only at the phase $\theta=-\pi / 2$ or $\theta=\pi / 2$, resulting in propagation in the $X$-direction. From the momentum conservation, the energy release is divided into two radiations; at $\theta=$ $-\pi / 2$ to the $+X$-direction and at $\theta=\pi / 2$ to $-X$-direction. The single radiation has the following energy:

$$
E_{\gamma}=E_{\gamma}^{+}=E_{\gamma}^{-}=\frac{\Delta E}{2}=\frac{E_{s}}{4 \omega_{0}^{2}} \omega^{2} .
$$

Spacias, in which energy is circulating in $H-X$ at $\omega_{0}$, transport the vibration in $H$ at $\omega$ to $X$. Therefore, the propagation speed is equal to the circulating velocity of the spacia shown by

$$
v_{x}=\mu_{0} \omega_{0} .
$$

Two pieces of the energy move in $X$ as follows:

$$
\mathbf{X}^{+}=v_{x}\left(t-\frac{\pi}{2 \omega_{0}}\right)=\mu_{0}\left(\omega_{0} t-\frac{\pi}{2}\right), \quad \mathbf{X}^{-}=-v_{x}\left(t-\frac{\pi}{2 \omega_{0}}\right)=-\mu_{0}\left(\omega_{0} t-\frac{\pi}{2}\right) .
$$

The vibration $\omega$ in $Y$ is also kept but the amplitude gets larger due to propagation to surrounding spacias at the speed $\mu_{0} \omega_{0}$, whereas the speed during the rotation was $\mu_{0} \omega$. We get the following formulas for the distribution in $Y$. The amplitude in $Y$ is inversely proportional to the frequency $\omega$ :

$$
\begin{gathered}
\mathbf{Y}^{+}=\frac{\omega_{0}}{\omega} \mu_{0}(\sin \omega t-j \cos \omega t), \quad \mathbf{Y}^{-}=-\frac{\omega_{0}}{\omega} \mu_{0}(\sin \omega t-j \cos \omega t), \\
v_{c}=\mu_{y} \omega=\left(\mu_{0} \omega_{0} / \omega\right) \omega=\mu_{0} \omega_{0} .
\end{gathered}
$$

The energy distribution in $H$ is shown by

$$
\mathbf{H}^{+}=\mu_{0}(\sin \omega t-i \cos \omega t), \quad \mathbf{H}^{-}=-\mu_{0}(\sin \omega t-i \cos \omega t) .
$$

Let us express the momentum in $H$ as the electric charge. If the vibrations in $H-X$ were in circle, the momentums $\mathbf{p}_{0}(-\pi / 2<\theta \leq \pi /)$ and $\mathbf{p}_{\pi}(\pi / 2<\theta \leq 3 \pi / 2)$ would be time-invariant at each position of $X$. In this case, the energy is vibrating only in $H$ with a linear movement in $X$, and the momentum in $H$ varies based on Eq. (6.23) as for $H-X$. The amplitude of the momentum vibration in $H$ is given as follows:

$$
p_{h}^{+}=p_{h}^{-}=p_{h}=\frac{E_{\gamma}(\omega)}{v_{c}}=\frac{E_{s} \omega^{2}}{4 \omega_{0}^{2} \mu_{0} \omega}=\frac{E_{s} \omega}{4 v_{x} \omega_{0}} .
$$


The circulating velocity in $H$ is

$$
\mathbf{v}_{\mathbf{h}}^{+}=\frac{d \mathbf{H}^{+}}{d t}=\mu_{0}(\cos \omega t+i \sin \omega t), \quad \mathbf{v}_{\mathbf{h}}^{-}=\frac{d \mathbf{H}^{-}}{d t}=-\mu_{0}(\cos \omega t+i \sin \omega t) .
$$

Take the initial direction of $p_{h}^{+}$at $t=0$ as $-i$ and that of $p_{h}^{-}$as $+i$. We get the following momentums in $H$ :

$$
\mathbf{p}_{\mathbf{h}}^{+}=-i p_{h}(\cos \omega t+i \sin \omega t), \quad \mathbf{p}_{\mathbf{h}}^{-}=i p_{h}(\cos \omega t+i \sin \omega t) .
$$

According to the definition of the electric charge by Eq. (6.7), i.e., $\mathbf{e} \equiv i \mathbf{p}$, we get the following electric charges:

$$
\begin{aligned}
& \mathbf{e}_{\gamma}^{+}=i \mathbf{p}_{\mathbf{h}}^{+}=p_{h}(\cos \omega t+i \sin \omega t)=e_{\gamma}(\cos \omega t+i \sin \omega t), \\
& \mathbf{e}_{\gamma}^{-}=i \mathbf{p}_{\mathbf{h}}^{-}=-p_{h}(\cos \omega t+i \sin \omega t)=-e_{\gamma}(\cos \omega t+i \sin \omega t) .
\end{aligned}
$$

The amplitude of the electric charges becomes

$$
e_{\gamma}=p_{h}=\frac{E_{s}}{4 v_{x} \omega_{0}} \omega
$$

As we see so far, the radiation is the "light". The phase velocity is the light speed $c$ :

$$
c=v_{x}=\mu_{0} \omega_{0} .
$$

Having the initial phase as $\pi / 2$, we get the following energy distribution of the light:

$$
\begin{gathered}
\mathbf{X}=c t=\mu_{0} \omega_{0} t \\
\mathbf{Y}=\frac{\omega_{0}}{\omega} \mu_{0}(\sin (\omega t+\pi / 2)-j \cos (\omega t+\pi / 2)) \\
=\frac{\omega_{0}}{\omega} \mu_{0}(\cos \omega t+j \sin \omega t)=\frac{\omega_{0}}{\omega} \mu_{0} \exp (j \omega t), \\
\mathbf{e}_{\gamma}=e_{\gamma}(\cos (\omega t+\pi / 2)+i \sin (\omega t+\pi / 2))=e_{\gamma} \exp (i(\omega t+\pi / 2)) .
\end{gathered}
$$

The respective propagations in $X$ of the electric charge and the energy location in $Y$ are expressed as follows:

$$
\begin{gathered}
e_{\gamma} \varphi_{e}=e_{\gamma} \exp (i(k x-(\omega t+\pi / 2))), \\
Y \varphi_{y}=\frac{\omega_{0}}{\omega} \mu_{0} \exp (j(k x-\omega t)), \\
k=\frac{\omega}{c}, \quad e_{\gamma}=\frac{E_{s}}{4 c \omega_{0}} \omega .
\end{gathered}
$$

There is the following relation between the energy and the amplitude of the electric charge:

$$
E_{\gamma}=\frac{4 c^{2}}{E_{s}} e_{\gamma}^{2} .
$$

When a molecule absorbs light, it incorporates one cycle of $H$ vibration of the light in its own quantized circulation in $X-Y$ of the $H$ vibration, resulting in excitation to 
the next energy level. For this reason, let us take the following property of the energy per cycle. There are $\omega /(2 \pi)$ cycles of $H$ vibration per second (unit time):

$$
E_{\gamma} / \text { cycle }=\frac{E_{s} \pi}{2 \omega_{0}^{2}} \omega=\frac{E_{s} \pi^{2}}{\omega_{0}^{2}} \nu .
$$

It should be the well-known "energy quantum" $E=\hbar \omega=h \nu$. We generally regard that the energy of a photon is $E=\hbar \omega=h \nu$. However, it is not per common time interval but per cycle. Transversely, we may define the "photon" as a single cycle of light. We get the following formulas for the respective energies of light and photon as well as the Planck's constant:

$$
\begin{gathered}
E_{\gamma}=\frac{E_{s}}{4 \omega_{0}^{2}} \omega^{2}=\frac{\hbar}{2 \pi} \omega^{2}=h \nu^{2} \quad \text { (light), } \\
E_{p}=\frac{E_{\gamma}}{\nu}=\hbar \omega=h \nu \quad \text { (photon) } \\
h=\frac{E_{s} \pi^{2}}{\omega_{0}^{2}} \quad \text { (Planck's constant) }
\end{gathered}
$$

In cosmological observation, the measured energy of light based on photons is multiplied by $1 /(1+z)$ because of the dilation of wavelength width by a redshift as a part of $K$-correction to convert the measuring frequency of light. ${ }^{8,19,20}$ This modification corresponds to the conversion of photon energy to the light energy. The integrated photon energy for one second (multiplying by $c / \lambda=\nu$ ) is the light energy.

If the added energy to the photino is high enough to rotate it at $\omega=\omega_{0}$, the rotating photino gets stable as a stationary particle, whose energy is quantized in one hidden $H$-dimensional and two space $X$ - and $Y$-dimensions.

\section{Light Speed Variation by the Space Expansion}

The light speed is the circulating velocity of the spacia $c=\mu_{0} \omega_{0}$. Along with the space expansion the density of the space energy decreases but the radius of the spacia in the hidden $H$-dimension remains constant as $\mu_{0}$ since the space expansion is only in the space dimensions. Hence the energy $E_{\mu}$ of the spacia decreases and the number of spacias increases by the space expansion. Express parameters as a function of the radius $x$ of the $4 \mathrm{D}$ sphere. The energy of the spacia, equal to that of the spacino, is given by

$$
E_{s}(x)=E_{\mu}(x)=E_{\mu} \frac{x_{p}^{3}}{x^{3}} \quad\left(x_{p}=\text { present radius }\right)
$$

Provide that the Planck's constant is invariant by the space expansion. The energy of light shows an invariant frequency $\omega$ at emission at any point of the space expansion:

$$
E_{\gamma}=\frac{E_{s}}{4 \omega_{0}^{2}} \omega^{2}=\frac{E_{s}(x)}{4\left(\omega_{0}(x)\right)^{2}} \omega^{2}=\frac{x_{p}^{3}}{x^{3}} \frac{E_{s}}{4\left(\omega_{0}(x)\right)^{2}} \omega^{2} .
$$


For the equation to be valid for any $E_{s}$ and $\omega$, the frequency of the spacino and the spacia varies by $x$ as follows:

$$
\left(\omega_{0}(x)\right)^{2}=\omega_{0}^{2} \frac{x_{p}^{3}}{x^{3}}
$$

The light speed is then given by

$$
c(x)=\mu_{0} \omega_{0}(x)=\frac{K_{1}}{\sqrt{x^{3}}} \quad\left(K_{1}=\text { a constant }\right) .
$$

This formula is by the original time $\tau$ since the initial value of the frequency $\omega_{0}\left(\mu_{0}\right)$ is based on $\tau$. Because the Observed Time is suitable to handle the redshift, light propagated distance and so on for cosmological expansion, derive the equation to that with the Observed Time $T$ equal to the radius $x$ as formerly reported. ${ }^{9}$ The expansion speed of $x$ by the original time $\tau$ is given by Eq. (4.22) if we use the Cosmic Unit for $x$ as one for the maximum radius of the universe:

$$
\frac{d x}{d \tau}= \pm \sqrt{2 K_{f} E_{U}\left(\frac{1}{x}-1\right)} .
$$

The fundamental force constant $K_{f}$ and the energy of the whole universe $E_{U}$ remain constant. From Eqs. (7.4) and (4.22) the light speed by the Observed Time becomes

$$
\begin{gathered}
C(x)=c(x) \frac{d \tau}{d x}=\frac{K_{1}}{\sqrt{2 K_{f} E_{U} x^{3}\left(\frac{1}{x}-1\right)}}, \\
C(x)=\frac{K}{x \sqrt{1-x}}, \quad \mu_{0}<x<1, K=\text { a constant. }
\end{gathered}
$$

This formula is exactly the same as the "energy density factor" $f_{D}$ of the light speed, which we formerly reported from the analogy of the sound speed in the air. ${ }^{5,9}$

Let us check the invariance of Planck's constant in $E_{\gamma}=h \nu^{2}$ and $E_{p}=h \nu$ by the space expansion while the circulating velocity (light speed) in $E=m v_{c}^{2}$ varies. The intrinsic energy $m_{\mu}$ of the spacia for the internal circulation, which exhibits the energy of the spacia $E_{\mu}=m_{\mu} v_{c}^{2}=m_{\mu} \mu_{0}^{2} \omega_{0}^{2}$, is a quantized energy circulation in other hidden dimensions. Because it is quantized, only specific skipping values are allowed. Thus, the intrinsic energy $m_{\mu}$ cannot vary but the circulating speed decreases along with the continuous space expansion. The Planck's constant is given as follows from Eq. (6.39):

$$
h=\frac{E_{s} \pi^{2}}{\omega_{0}^{2}}=\frac{m_{\mu} \mu_{0}^{2} \omega_{0}^{2} \pi^{2}}{\omega_{0}^{2}}=m_{\mu} \mu_{0}^{2} \pi^{2} \quad \text { (Planck's constant). }
$$

Because $m_{\mu}$ and $\mu_{0}$ are invariant, the Planck's constant $h$ is invariant. The decrease of $\omega_{0}$ is similar to the redshift of light by the space expansion. In the case of an energy separation such as the Giant Separation, the intrinsic energy is divided into two pieces with the frequency $\omega_{0}$ being unchanged. However, in a space expansion, the intrinsic energy remains unchanged and the frequency decreases. 


\section{Electric Charge Separation and Electric Force}

\subsection{Electric force}

The energy circulation in the hidden dimension $H$ and a space direction $(X)$ has a special property that it can spread to surrounding spacias with keeping the original vibration in $H$. The quantized circulation in $H-X$ in a single spacia is the photino and has an electric charge polarization as discussed in Sec. 6.1. The polarized electric charges of the photino are given as follows for the respective arcs $-\pi / 2<\theta \leq \pi / 2$ (collectively referred to as $\theta=0$ ) and $\pi / 2<\theta \leq 3 \pi / 2$ (collectively referred to as $\theta=\pi$ ), where $E_{s}$ denotes the energy of the spacino. The energy of the photino is $E_{s} / 2$ :

$$
\mathbf{e}_{\mathbf{p}}^{-} \equiv \mathbf{e}_{\mathbf{0}}=-p_{h}=-\frac{E_{s}}{4 v_{c}}, \quad \mathbf{e}_{\mathbf{p}}^{+} \equiv \mathbf{e}_{\pi}=p_{h}=+\frac{E_{s}}{4 v_{c}} .
$$

The electric force between the two arcs is given as follows:

$$
F_{p}=K_{f} \frac{\mathbf{e}_{\mathbf{p}}^{-} \cdot \mathbf{e}_{\mathbf{p}}^{+}}{\left(2 \mu_{0}\right)^{2}} .
$$

In a single spacia there can exist a photino and another quantized component consisting of one or plural circulations in only space dimensions. As an example, take a quantized particle containing a pair of conjugate circulations in two space dimensions $X$ and $Y$ and a circulation in the hidden $H$ - and space $X$-dimensions. It is a fusion particle of an $S_{0}$ spacino and a photino. Each circulation has the energy $E_{s} / 2$ and the total energy is $3 E_{s} / 2$. Refer the particle to as the "electric dipole", which has the following wave functions:

$$
\begin{aligned}
& \frac{3 E_{s}}{2} \phi_{d}=\frac{E_{s}}{2}\left(\varphi_{x y}+\varphi_{x y}^{*}\right)+\frac{E_{s}}{2} \varphi_{x}, \\
& \varphi_{x}=\exp \left(i \omega_{0} t\right), \quad \mathbf{X}=\mu_{0} \varphi_{x}, \quad \mathbf{H}=-i \mu_{0} \varphi_{x}, \\
& \varphi_{x y}=\exp \left(j \omega_{0} t\right), \quad \mathbf{X}=\mu_{0}\left(\varphi_{x y}+\varphi_{x y}^{*}\right), \quad \mathbf{Y}=-j \mu_{0}\left(\varphi_{x y}+\varphi_{x y}^{*}\right) .
\end{aligned}
$$

Apply a force and separate the conjugate $X-Y$ circulations $\varphi_{x y}$ and $\varphi_{x y}^{*}$ from each other in the $X$-direction by $r$ with energy being added. Along with the separation, the $H-X$ circulation is prolonged. Similar to the light propagation, from the spacia carrying $\varphi_{x y}$ the energy vibration in $H$ propagates to the next spacias in the $X$-direction towards the spacia carrying $\varphi_{x y}^{*}$ together with the reverse phenomenon from $\varphi_{x y}^{*}$ to $\varphi_{x y}$. This is an energy exchange between the two end spacias, where the respective electric charges remain the same as before the exchange. Between the two charges there works the following electric force:

$$
\mathbf{F}_{\mathbf{p}}(r)=K_{f} \frac{\mathbf{e}_{\mathbf{p}}^{-} \cdot \mathbf{e}_{\mathbf{p}}^{+}}{r^{2}}=-K_{f} \frac{e_{p}^{2}}{r^{2}}
$$

We may interpret the force alternatively as the $X-H$ circulation spreads to next spaces. The spacia at $\varphi_{x y}$ rotates the next spacia in $H-X$ by $-\omega_{0}$, which further 
rotates the next one by $\omega_{0}$, and the process repeats until the spacia at $\varphi_{x y}^{*}$. The electric charges line up reciprocally as $(-+)(+-)(-+) \cdots(+-)(-+)$. The minus (attractive) electric force works within a spacia to accelerate energy movements centripetally to circulation. The plus (repulsive) electric force works for the adjacent spacias to separate and rotate each other. Let $n$ (odd number) be the number of spacias for the $H-X$ circulations. The distance between the plus end and the minus end is $r=2 n \mu_{0}$. The energy of the initial circulation is divided into $n$ circulations. Therefore, the energy of a single circulation in a single spacia becomes

$$
E_{p}(r)=\frac{E_{p}}{n}=E_{p} \frac{2 \mu_{0}}{r} .
$$

The intrinsic energy is divided into $n$ circulations, and the circulating velocity $v_{c}=$ $\mu_{0} \omega_{0}$ does not change. Thus the electric charge in a single spacia becomes

$$
e_{p}(r)=p_{h}(r)=\frac{e_{p}}{n}=e_{p} \frac{2 \mu_{0}}{r} .
$$

Accordingly, the force within a single spacia is given by

$$
\mathbf{F}_{\boldsymbol{\mu}}=K_{f} \frac{\mathbf{e}_{\mathbf{p}}^{-}(r) \cdot \mathbf{e}_{\mathbf{p}}^{+}(r)}{\left(2 \mu_{0}\right)^{2}}=-K_{f} \frac{e_{p}^{2}}{r^{2}} .
$$

In total, there appear the force at $\theta=0$ of the spacia at $\varphi_{x y}$ and that at $\theta=\pi$ of the spacia at $\varphi_{x y}^{*}$, exhibiting the same Eq. (8.4). The forces between the two points are set off.

\subsection{Electrically charged particles and electric current}

There can be various adducts of a photino with quantized circulations in space dimensions other than an $S_{0}$ spacino mentioned above. Each charged end spacia can carry various numbers of quantized circulations in up to three planes orthogonal to each other of space dimensions. They are an electrically charged quantum particle. The prolongation of the electric dipole shown by Eqs. (8.3) would give the pair of respective precursors of electron and positron. Such a precursor would separate to an electron (or positron) and a neutrino. The electric force is common for such particles of various energies but depends only on the electric charge. The electric charge $\mathbf{e}_{p}^{-}$or $\mathbf{e}_{\mathbf{p}}^{+}$of the photino shown by Eq. (8.1) is the "elementary charge". Charged particles should make a pair of plus and minus $e_{p}$ as an $H-X$ energy circulation while the pair can be prolonged. The prolongation of an electric pair is possible, for instance, by a circulation of the pair seen in an atom or by a force from a neighboring electric charge. Here, let us use the term "photino" as any photino component associated with any masses at two charge ends. When plural photinos align, a pair exchange with recircularization can occur. Take a chain of plural photinos. Lay a negative charge next to the left plus end of the chain. Sequential electric pair exchanges show a negative charge movement from the left to the right. We can also regard the process 
alternatively that a positive charge moves from the right to the left because the real nature is the pair exchange. The process results in the "electric current". The electric current is not the movement of the whole electron including the energy circulation in two space dimensions but the movement of only the electric charge. A negative side of a photino in an atom or a molecule is an electron. A molecule containing a photino can relay the electric pair exchange, i.e., it is electrically conductive.

\subsection{Magnetism}

As an example of a series of photinos, each of which has paired electric charges, take two photinos $(+-)$ and $(+-)$ in the space $X$-direction. Number the locations of electric charge and mass as 1,2,3 and 4 . Bring a negative elementary charge $e_{p}^{-}$close to the location 1 from the left as shown in Fig. 3. An electric pair exchange happens and gives a plus-minus pair at 1 and a minus charge at 2 . The next pair exchange gives a recovered pair (+-) between 1 and 2, a plus-minus pair at 3 and a minus charge at 4 . A further pair exchange gives a minus charge to the right from 4 with the initial photino chain from 1 to 4 . A plus-minus pair at a single location in $X$ at 1 or 3 is in the plane $Y-Z$ orthogonal to $X$. The intrinsic energy is circulating in $H-Y Z$, which results in electric polarization in $Y-Z$. Because the electric charges can transmit to next spacias, the electric pair can rotate around $X$ in $Y-Z$ in addition to prolongation of the radius in $Y-Z$. The speed of the electric charge movement in $Y-Z$ is the light speed because the movement is mediated by rotation of spacias. This is a rotation of a prolonged electric pair, equal to a light circulation, around $X$ at a single location of $X$ such as 1 and 3 .

The electric pair exchange in $X$ itself does not transmit the energy. An added electric energy can transmit in a chain of electric pairs as an energy circulation

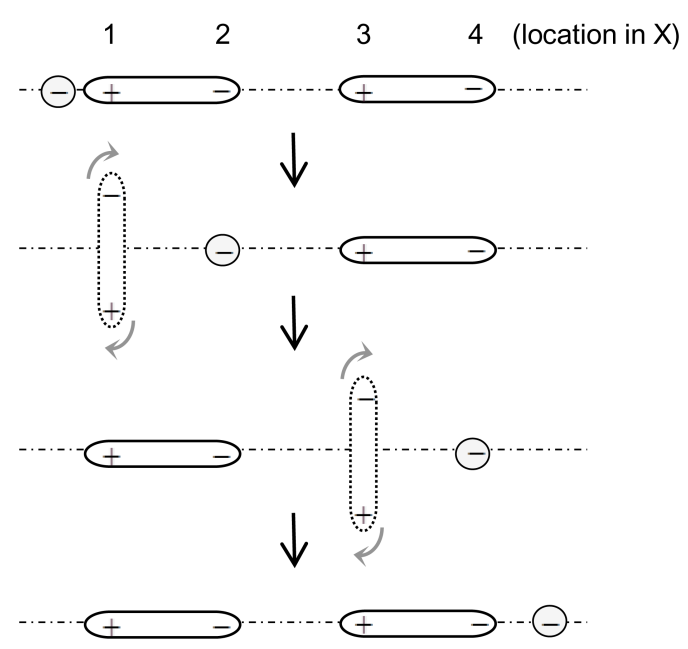

Fig. 3. Electric current and magnetism. 
around the chain. Each position on the circumference of an energy circulation has its own momentum vector. Between two momentums the force given by Eq. (2.8) works as follows:

$$
F=K_{f} \frac{p_{1} p_{2}}{d^{2}} \cos \theta_{p} \sin \theta_{1} \sin \theta_{2}=K_{f} \frac{\mathbf{p}_{\mathbf{1}} \cdot \mathbf{p}_{\mathbf{2}}}{d^{2}} \sin \theta_{1} \sin \theta_{2}
$$

Similar to the electric charge let us define the "magnetic charge" as follows:

$$
\mathbf{b} \equiv \mathbf{p}
$$

We get the following formula for the force between magnetic charges:

$$
F=K_{f} \frac{\mathbf{b}_{\mathbf{1}} \cdot \mathbf{b}_{\mathbf{2}}}{d^{2}} \sin \theta_{1} \sin \theta_{2}=K_{f} \frac{b_{1} b_{2}}{d^{2}} \cos \theta_{p} \sin \theta_{1} \sin \theta_{2}
$$

The magnetic charge is a momentum in a space direction whereas the electric charge is a momentum in the hidden $H$-dimension. The magnetic charge is a vector without a specific standard direction. The relativity of directions affects the force.

The added energy becomes the energy of the electric current. However, it is not an energy movement in the current line in $X$ but an energy circulation around the current. There is a flexibility in the combination of frequency and radius of the $Y-Z$ circulation. A magnetic circulation does not have to be a pure circle but can change the shape by outer influence as far as keeping a circulation. A rotation of a photino around a space axis induces a pair of opposite linear propagations of light. A linear movement of a photino (electric charge) in a space direction presents a circulation of light around the movement:

\section{Conclusion}

Starting from the premise that the initial energy is vibrations in multiple dimensions, we proposed the energy circulation theory. Movement of an intrinsic energy vests an additional energy and exhibits a new total energy. The total energy can work as a new intrinsic energy for the direction perpendicular to the original movement. The fundamental force works between energy movements as shown by Eq. (2.8). Its charge is the momentum, a one-dimensional charge, in contrast to the fact that the charge of the gravity is the energy, a zero-dimensional charge. The momentum in a hidden dimension is especially referred to as the electric charge, the force by which is the electric force. A minus force is attractive and works on the energy pieces causing them to bend and make a single circle.

A movement at $v$ of an intrinsic energy $m$ shows the energy $E=m v^{2}$, which we call the dynamic energy. For a perpendicular direction to the original movement, the dynamic energy works as a rest energy. The dynamic energy from an energy circulation can be treated as quantized and works as the rest energy for any direction. Under the common energy circulating velocity (light speed), the rest energy $E_{0}$ and the intrinsic energy $m_{0}$ are in proportion as shown by $E_{0}=m_{0} v_{c}^{2}=m_{0} c^{2}$. 
We usually use the intrinsic energy of the internal circulation as a mass for further motion instead of the rest energy.

Based on the energy circulation theory, we showed an evolution to a $4 \mathrm{D}$ spherical universe. Simultaneous separation of two pairs of conjugate energy circulations gave two universes with an energy distribution in a 3D surface of a 4D sphere. The energy distribution is expanding in four dimensions whereas movements in other dimensions are quantized in a steady state. The lowest frequency $\omega_{0}$ and the longest radius $\mu_{0}$ of the hidden dimensions play an important role. The energy movement of the universe is expressed by Eq. (4.9) in the 4D space and by Eq. (4.14) in the 3D space. We can divide the energy into two parts. The space energy is the rest energy corresponding to the vacuum space. The apparent energy is the kinetic energy and what we detect as energy in the $3 \mathrm{D}$ space. We referred the $3 \mathrm{D}$ ball area of radius $\mu_{0}$ to as the unit space and the space energy in the area to as the spacia. A spacia can carry an apparent energy by its rotation and transmit it to the next spacias. We can express an apparent energy as a vibration of the space energy.

An excitation of a spacia presents a quantum particle, in which an intrinsic energy circulates at the circulating velocity $v_{c}=\mu_{0} \omega_{0}$. We named an unconjugated single energy circulation in one hidden and one space dimensions as the photino. The momentum in the hidden dimension is the electric charge defined by Eq. (6.7). There is an electric charge polarization in a photino, which can be prolonged in a space direction mediated by spacias. We derived the equation of the electric force Eq. (8.4) from the fundamental force. A rotation of a photino radiates light as shown by Eqs. (6.30)-(6.32). We demonstrated the light energy to be Eq. (6.37), the photon energy to be Eq. (6.38) and the Planck's constant to be Eq. (6.39). The light speed is the energy circulating velocity in the spacia $c=v_{c}=\mu_{0} \omega_{0}$. A linear movement of a photino (electric charge) presents a circulation of light around the movement (electric current). The momentum of the energy circulation in a space direction is the magnetic charge.

It is surprising to us that the energy circulation theory has induced the $4 \mathrm{D}$ spherical universe and it can explain such important unresolved issues in physics. One may complain that the fundamental force based on momentums would be tricky. However, it is the more fundamental nature of the electric and magnetic forces. An experimental evidence supporting the 4D spherical model is the Hubble diagrams of observed supernovae. We reported previously that the expected line from the model for the case that the present radius (Observed Time) is 0.7 times of its maximum $x=T_{p}=0.7$ showed an excellent overlap with the line of flat $\Omega_{m}=0.27 \Lambda$ CDM universe that Rubin et al. concluded as the best fit to the observed data of supernovae. $^{8,21}$

\section{Discussions}

In standard physics two principles are underlined; the light speed invariance and the rest-mass invariance from the variation of an observing frame. They are not 
theoretical ones but rather are assumptions, which are said to successfully explain real observations.

As we discussed in Sec. 1, the light speed invariance is not yet experimentally proved. If we abandon it, those experimental evidences for the Lorentz transformation of the special relativity turn to show a light speed variation. The interpretation of the Hubble diagram of observed supernovae that the expansion of the universe is accelerating is also based on the light speed invariance during the whole expansion period. According to the energy circulation theory, the light speed varies along with the space expansion as discussed in Sec. 7. The observed data of supernovae imply that the radial expansion speed of the universe is decelerating as expressed by Eq. (4.22) by the original time and is constant by the Observed Time as shown by Eq. (4.23). It is not a matter whether we use the original time or the Observed Time, but the ECT projects a specific correlation between the redshift and the brightness of stars, which nicely conformed to the observed data.

The rest-mass invariance by frame is also from the special relativity. Based on it, the general relativity insists the time delay by the gravity. Pound and Rebka reported $^{22}$ in 1960 the difference of the gravitational potential over a height of $22.5 \mathrm{~m}$ as a shift in frequency of the electromagnetic radiation of ${ }^{57} \mathrm{Fe}$. According to the general relativity, they interpret that the results come from the difference of the gravitational redshift by height, and claim that the time at a higher altitude passes faster than that at a lower altitude provided that the rest mass is kept invariant. However, if we once abandon the rest-mass invariance, the results would imply the increase of the rest mass due to the increase of the gravitational potential at a higher altitude. We plan to report it in detail by a separate paper.

If the rest-mass invariance is invalid, we have to define the term "mass", which varies by a frame. In ECT, mass is defined as the quantity of an intrinsic energy and several variations of mass, frame and direction are given in Sec. 2.2. We usually regard that the resistance for acceleration by a force is the rest mass. According to ECT, the rest energy should primarily be the resistance. However, we can use rest mass instead of rest energy because they are in proportion under the common circulating velocity of internal motion equal to the light speed.

Another notable difference of ECT from the standard cosmology is that the main force, which regulates the space expansion, is the fundamental force working on momentums rather than the gravitational force. We formerly expected that the force would be the gravity. However, the gravity seems too weak to give early stages of the universe. The fundamental force between a huge energy center subject and a small energy circulating subject is very small and the gravitational interaction is dominant. However, between individual energy components circulating on a circle, the fundamental force should be more remarkable than the gravity. In the case of space expansion, the energy circulating velocity of the universe is extremely huge as shown in Eq. (4.11) exhibiting huge momentums.

The 4D spherical model from ECT proposes that the vacuum space has the space energy, vibrations of which show our observable energies (apparent energies) in the 
3D space. In the standard cosmology, currently various candidates for the dark energy are proposed to cause the acceleration of the cosmic expansion. They are not a medium for observable energies but something exhibiting a repulsive force (negative pressure). If we define the term "dark energy" simply as an unknown energy, we may see the space energy as a dark energy. However, the term "space energy" is intended to directly imply the energy of the space. Lastly, we hope that someone would check the potential circadian rhythm in the brightness of the combined beam by a latest Michelson interferometer for detecting gravitational waves. It would imply an anisotropy of the light speed by the frame attached to the apparatus, which signifies the existence of a medium for the light propagation.

\section{References}

1. S. Perlmutter et al., Measurements of the cosmological parameters $\Omega$ and $\Lambda$ from the first seven supernovae at $z \geq 0.35$, Astrophys. J. 483 (1997) 565-581.

2. S. Perlmutter et al., Discovery of a supernova explosion at half the age of the universe, Nature 391 (1998) 51-54.

3. S. Perlmutter et al., Measurements of $\Omega$ and $\Lambda$ from 42 high-redshift supernovae, Astrophys. J. 517 (1999) 565-586.

4. B. Schmidt et al., The high- $Z$ supernova search: Measuring cosmic deceleration and global curvature of the universe using type Ia supernovae, Astrophys. J. 507 (1998) 46-63.

5. S. Nagao, Light propagated distance and redshift of a distant star, J. Phys., Conf. Ser. 626 (2015) 012068.

6. S. Nagao, Acceleration factor for propagation of a stationary wave in its wave medium: Movement of energy in the 3-D space, J. Phys., Conf. Ser. 442 (2013) 012067.

7. Wikipedia, Copenhagen interpretation (2018), https://en.wikipedia.org/wiki/Copenhagen_ interpretation.

8. S. Nagao, Frequency-based redshift for cosmological observation and Hubble diagram from the 4-D spherical model in comparison with observed supernovae, J. Phys., Conf. Ser. 880 (2017) 012058.

9. S. Nagao, Light speed and the expansion of the universe, J. Phys., Conf. Ser. 306 (2011) 012073 .

10. S. Nagao, Nature of tracing dimension, imaginary order of freedom and our observed time passing at constant speed, J. Phys., Conf. Ser. 174 (2009) 012069.

11. A. A. Michelson, The relative motion of the earth and the luminiferous ether, Am. J. Sci. 22 (1881) 120-129.

12. A. A. Michelson and E. Morley, On the relative motion of the earth and the luminiferous ether, Am. J. Sci. 34 (1887) 333-345.

13. S. Hermann et al., Rotating optical cavity experiment testing Lorentz invariance at the 10 ${ }^{-17}$ level, Phys. Rev. D 80 (2009) 105011, arXiv:1002.1284 [physics.class-ph].

14. A. Einstein, Zur elektrodynamik bewegter körper, Ann. Phys. 322 (1905) 891-921. [English translation: http://www.fourmilab.ch/etexts/einstein/specrel/www/].

15. A. Einstein, Ist die Trägheit eines Körpers von seinem Energieinhalt abhängig? Ann. Phys. 323 (1905) 639-643 [English translation: http://www.fourmilab.ch/etexts/ einstein/E_mc2/www/].

16. Wikipedia, Special relativity (2018), https://en.wikipedia.org/wiki/Special_relativity.

17. Wikipedia, Mass-energy equivalence (2018), https://en.wikipedia.org/wiki/Mass\%E2\% 80\%93energy_equivalence. 
18. S. Sunakawa, in Sotaisei Riron no Kangaekata (Vision of the Theory of Relativity) (Iwanami Shoten, Tokyo, 1993), pp. 56-58.

19. P. Nugent et al., $K$-corrections and extinction corrections for type Ia supernovae, $P u b l$. Astron. Soc. Pac. 114 (2002) 803-819.

20. V. de Lapparent et al., The ESO-sculptor survey: Luminosity functions of galaxies per spectral type at redshifts 0.1-0.5, Astron. Astrophys. 404 (2003) 831-860.

21. D. Rubin et al., Precision measurement of the most distant spectroscopically confirmed supernova Ia with the Hubble space telescope, Astrophys. J. 763 (2013) 35-44.

22. R. V. Pound and G. A. Rebka, Jr., Apparent weight of photons, Phys. Rev. Lett. 4 (1960) $337-341$. 